TRANSACTIONS OF THE

AMERICAN MATHEMATICAL SOCIETY

Volume 349, Number 12, December 1997, Pages 4787-4814

S $0002-9947(97) 01997-1$

\title{
A THEOREM OF THE DORE-VENNI TYPE FOR NONCOMMUTING OPERATORS
}

\author{
SYLVIE MONNIAUX AND JAN PRÜSS
}

\begin{abstract}
A theorem of the Dore-Venni type for the sum of two closed linear operators is proved, where the operators are noncommuting but instead satisfy a certain commutator condition. This result is then applied to obtain optimal regularity results for parabolic evolution equations $\dot{u}(t)+L(t) u(t)=f(t)$ and evolutionary integral equations $u(t)+\int_{0}^{t} a(t-s) L(s) u(s) d s=g(t)$ which are nonautonomous. The domains of the involved operators $L(t)$ may depend on $t$, but $L(t)^{-1}$ is required to satisfy a certain smoothness property. The results are then applied to parabolic partial differential and integro-differential equations.
\end{abstract}

\section{INTRODUCTION}

Let $X$ be a Banach space with norm $|\cdot|$, and let $A$ be a closed linear operator in $X$ with dense domain $D(A)$; as usual, $N(A), R(A), \rho(A), \sigma(A)$ denote kernel, range, spectrum, resolvent set of $A$, respectively. $A$ is called sectorial if $N(A)=\{0\}, R(A)$ is dense in $X, \rho(A) \supset(-\infty, 0)$, and $M_{0}:=\sup _{r>0} r\left|(r+A)^{-1}\right|<\infty$. The class of sectorial operators will be denoted by $S(X)$. If $A$ is sectorial it follows easily that $\rho(-A)$ contains a nonempty open sector $\Sigma_{\phi}:=\{z \in \mathbb{C}: z \neq 0,|\arg z|<\phi\}$. Therefore, for such operators it makes sense to define the spectral angle $\phi_{A}$ of $A$ by means of

$$
\phi_{A}:=\inf \left\{\phi>0: \rho(A) \supset-\Sigma_{\pi-\phi}, M_{\pi-\phi}<\infty\right\},
$$

where $M_{\phi}:=\sup \left\{\left|\lambda(\lambda+A)^{-1}\right|: \lambda \in \Sigma_{\phi}\right\}$. Obviously,

$$
\pi>\phi_{A} \geq \arg (\sigma(A)):=\sup \{|\arg \lambda|: \lambda \neq 0, \lambda \in \sigma(A)\} .
$$

Now given two sectorial operators $A$ and $B$ which are commuting in the sense that

$$
(\lambda+A)^{-1}(\mu+B)^{-1}=(\mu+B)^{-1}(\lambda+A)^{-1}, \quad \text { for all } \lambda \in \rho(-A), \mu \in \rho(-B),
$$

about 20 years ago, Da Prato and Grisvard [8] proved that the sum $A+B$ with (natural) domain $D(A+B):=D(A) \cap D(B)$ is densely defined, closable, and its closure $L$ is again sectorial with $\phi_{L} \leq \max \left\{\phi_{A}, \phi_{B}\right\}$, provided the parabolicity assumption $\phi_{A}+\phi_{B}<\pi$ is satisfied.

Received by the editors May 22, 1995.

1991 Mathematics Subject Classification. Primary 47A60, 47B47, 47G20, 47D06; Secondary 45A05, 45D05, 45K05.

Key words and phrases. Sum of linear operators, bounded imaginary powers of linear operators, commutator conditions, parabolic evolution equations, parabolic evolutionary integral equations, completely positive kernels, fractional derivatives, creep functions, viscoelasticity.

(C) 1997 American Mathematical Society 
The natural question arising in this context is whether $A+B$ is already closed, i.e. $L=A+B$. Da Prato and Grisvard [8] were able to show the latter in certain special cases when $X$ is a Hilbert space, but in general $A+B$ need not be closed, as was pointed out by Baillon and Clément [3]. A positive answer was given by Dore and Venni [9], even for non-Hilbert spaces.

To describe their result, observe that for the class of sectorial operators one can define complex powers by means of the standard Dunford integral; these will be closed linear and densely defined, but unbounded in general. A sectorial operator $A$ is said to admit bounded imaginary powers if the purely imaginary powers $A^{i s}$ of $A$ are uniformly bounded for $s \in[-1,1]$. It then can be shown that $A^{i s}$ forms a strongly continuous $C_{0}$-group of bounded linear operators. The class of such $A$ will be denoted by $B I P(X)$. The type $\theta_{A}$ of the $C_{0}$-group $A^{\text {is }}$ is called the power angle of $A$, i.e. $\theta_{A}:=\lim _{|s| \rightarrow \infty}|s|^{-1} \log \left|A^{i s}\right|$. The inequality $\theta_{A} \geq \phi_{A}$ has been proved in Prüss and Sohr [17].

Assuming that the Banach space $X$ is of class $\mathcal{H} \mathcal{T}$ (see Section 2), that $A$ and $B$ are commuting and admit bounded imaginary powers, and that the strong parabolicity condition $\theta_{A}+\theta_{B}<\pi$ is satisfied, the Dore-Venni theorem in the extended version obtained by Prüss and Sohr [17] states that $A+B$ is closed, sectorial, admits bounded imaginary powers, and $\theta_{A+B} \leq \max \left\{\theta_{A}, \theta_{B}\right\}$.

Both, the Da Prato-Grisvard theorem and the Dore-Venni theorem have important applications to evolution equations and to evolutionary integral equations, as has been shown in many articles. By means of these results maximal regularity properties of such problems can be proved, which are of interest not only in the linear theory but in particular for nonlinear problems.

However, the commutativity assumption appears to be fairly restrictive. Consider for example an evolution equation of the form

$$
\dot{u}(t)+L(t) u(t)=f(t), \quad t \geq 0, \quad u(0)=0,
$$

in a Banach space $X$, where $L(t)$ is a family of sectorial but generally unbounded operators in $X$. The commutativity assumption then requires the family $L(t)$ to be independent of $t$. Therefore it is very desirable to weaken this condition. For the Da Prato-Grisvard theorem it is well known how to do this. Already Da Prato and Grisvard [8] themselves presented a condition on the commutator of $B$ and $(\lambda+A)^{-1}$ such that their result still holds, and later their condition was replaced by a different, more flexible one by Labbas and Terreni [14]; see also Fuhrmann [13]. For the Dore-Venni theorem such extensions are still missing, at present.

It is the purpose of this paper to close this gap. Our main result shows that the Dore-Venni theorem remains valid in case $A$ and $B$ do not commute but are subject to a commutator condition of the Labbas and Terreni type. The proof is based on the techniques developed by Dore and Venni [9] and by Prüss and Sohr [17], combined with new estimates resulting from the Labbas-Terreni condition involving the complex powers of $A$ and $B$.

Our main result yields new maximal regularity results, for evolution equations as well as for evolutionary integral equations of the form

$$
u(t)+\int_{0}^{t} a(t-s)(L(s) u(s)-f(s)) d s=0, \quad t \geq 0,
$$

where $a(t)$ is a scalar kernel and $L(t)$ is as before. For the case of parabolic evolution equations (1.3) this is a straightforward extension of the work of Acquistapace and 
Terreni [2]; therefore the presentation is kept concise at that point, here. On the other hand, for the evolutionary integral equation (1.4) the application of the main result is not obvious; it involves new $L^{1}$-estimates for the solutions of linear scalar Volterra equations depending on a parameter.

The plan for this paper is as follows. In Section 2 the main results about the sum $A+B$ of two closed linear operators are stated and discussed, while the proofs are given in Section 4. Section 3 is devoted to the application of Theorem 1 and its corollaries to evolution equations (1.3) and evolutionary integral equations (1.4). Here emphasis is put on the interpretation of the commutator condition. The $L^{1}$-estimates for the resolvent kernel associated with $a(t)$ which are needed for the commutator condition are derived in Section 5. The paper concludes with applications to parabolic partial differential and integro-differential equations which are presented in Section 6 .

\section{The Main Results}

Recall that a Banach space $X$ is said to belong to the class $\mathcal{H} \mathcal{T}$ if the Hilbert transform $H$ defined by

$$
(H f)(t)=\lim _{\varepsilon \rightarrow 0+} \int_{|s| \geq \varepsilon} f(t-s) \frac{d s}{\pi s}, \quad t \in \mathbb{R}, f \in C_{0}^{\infty}(\mathbb{R} ; X),
$$

extends to a bounded linear operator on $L^{p}(\mathbb{R} ; X)$ for some $p \in(1, \infty)$. It is wellknown that the class $\mathcal{H} \mathcal{T}$ coincides with the class of Banach spaces having the uniform martingale difference property (UMD-spaces), and that for such spaces the Hilbert transform is bounded on $L^{p}(\mathbb{R} ; X)$ for every $p \in(1, \infty)$. In particular, Hilbert spaces belong to $\mathcal{H} \mathcal{T}$, and if $Y \in \mathcal{H} \mathcal{T}$ and $(\Omega, \mu)$ is a $\sigma$-finite measure space, then $L^{p}(\Omega, \mu ; Y)$ belongs to $\mathcal{H} \mathcal{T}$ for each $p \in(1, \infty)$. For a reference and further discussions we recommend the paper by Dore and Venni [9], the survey article Burkholder [6], and the monograph Prüss [16].

Consider now two closed linear densely defined operators $A$ and $B$ which are sectorial, admit bounded imaginary powers, and are subject to the strong parabolicity assumption $\theta_{A}+\theta_{B}<\pi$. Fix angles $\varphi_{A}>\theta_{A}, \varphi_{B}>\theta_{B}$, with

$$
\varphi_{A}+\varphi_{B}<\pi \text {. }
$$

Then there are constants $K_{A}, K_{B}$, such that

$$
\left|A^{i s}\right| \leq K_{A} e^{|s| \varphi_{A}}, \quad\left|B^{i s}\right| \leq K_{B} e^{|s| \varphi_{B}}, \quad \text { for all } s \in \mathbb{R} .
$$

Moreover, there is a constant $M_{B}$ such that

$$
\left|(\mu+B)^{-1}\right| \leq M_{B} /|\mu|, \quad \text { for all } \mu \in \Sigma_{\pi-\varphi_{B}} .
$$

A similar estimate is also valid for the resolvent of $A$, but if we assume in addition that $A$ is invertible, we have the stronger estimate

$$
\left|(\lambda+A)^{-1}\right| \leq M_{A} /(1+|\lambda|), \quad \text { for all } \lambda \in \Sigma_{\pi-\varphi_{A}},
$$

for some constant $M_{A}$. The commutator condition which will be employed here reads as follows. We assume that there are constants $0 \leq \alpha<\beta \leq 1$ and $c \geq 0$ such that

$$
\begin{array}{r}
\left|A(\lambda+A)^{-1}\left[A^{-1}(\mu+B)^{-1}-(\mu+B)^{-1} A^{-1}\right]\right| \leq \frac{c}{\left(1+|\lambda|^{1-\alpha}\right)|\mu|^{1+\beta}}, \\
\text { for all } \lambda \in \Sigma_{\pi-\varphi_{A}}, \mu \in \Sigma_{\pi-\varphi_{B}} .
\end{array}
$$


We are now in position to state our main result.

Theorem 1. Suppose $X$ is a Banach space of class $\mathcal{H} \mathcal{T}, A$ and $B$ are closed, linear, densely defined operators in $X$ which are sectorial and admit bounded imaginary powers, and let $A$ be invertible. Assume the strong parabolicity condition $\theta_{A}+\theta_{B}<$ $\pi$, and fix angles $\varphi_{A}>\theta_{A}, \varphi_{B}>\theta_{B}$, such that $\varphi_{A}+\varphi_{B}<\pi$ holds.

Then there is a constant $c_{0}>0$ such that the operator $A+B$ with domain $D(A) \cap D(B)$ is closed and invertible in $X$, provided $A$ and $B$ satisfy the commutator condition (2.6) and $c<c_{0}$.

The idea of the proof is based on the formulas

$$
S:=\frac{1}{2 i} \int_{\gamma-i \infty}^{\gamma+i \infty} A^{-z} B^{z-1} \frac{d z}{\sin (\pi z)}=\frac{1}{2 \pi i} \int_{\Gamma}(\mu+A)^{-1}(\mu-B)^{-1} d \mu,
$$

and

$$
T:=\frac{1}{2 i} \int_{\gamma-i \infty}^{\gamma+i \infty} B^{-z} A^{z-1} \frac{d z}{\sin (\pi z)}=\frac{1}{2 \pi i} \int_{\Gamma}(\mu-B)^{-1}(\mu+A)^{-1} d \mu,
$$

where $\gamma \in(0,1)$ is arbitrary, and the contour $\Gamma$ is chosen appropriately. One can then prove the identities

$$
A S x+S B x=x \quad \text { for all } x \in D(B),
$$

and

$$
T A x+B T x=x \quad \text { for all } x \in D(A) .
$$

Therefore, $A S$ and $S B$ are bounded or unbounded simultaneously, and the same is true for $T A$ and $B T$. The commutator condition (2.6) implies that $S B-B T$ is bounded; hence the operators $A S, S B, T A, B T$ are bounded or unbounded simultaneously. By means of the boundedness of the Hilbert transform in $L^{2}(\mathbb{R} ; X)$ and with the aid of (2.6) one then can show that all these quantities are bounded. This is the crucial step of the proof. Once this is done, one can construct left and right inverses $L$ and $R$ by means of the commutator condition (2.6) as

$$
L:=A^{-1}(I+Q)^{-1} A S, \quad R:=T A^{\gamma}(I+P)^{-1} A^{-\gamma},
$$

where $P$ and $Q$ are small in operator norm; it is here where the smallness of $c$ comes in. Finally, $R=L$ is an inverse, and since by construction $A L$ is bounded, $B L$ is bounded as well.

From the estimates derived in the proof in Section 4 it will become apparent that in the situation of Theorem $1 A+B$ will again be sectorial. More precisely, we have

Corollary 1. Let the assumptions of Theorem 1 be satisfied, in particular let $A$ and $B$ satisfy the commutator condition (2.6) with $c<c_{0}$.

Then $A+B$ is sectorial and $\phi_{A+B} \leq \max \left\{\varphi_{A}, \varphi_{B}\right\}$.

Since the basic estimates for the resolvents and the imaginary powers of $A$ and $B$ are basically invariant under shifts $\nu+A$ or $\nu+B$ where $\nu>0$ (cf. Prüss and Sohr [17]), but the constant $c$ in (2.6) decreases to zero if $\nu \rightarrow \infty$ after replacing $\beta$ by a slightly smaller and $\alpha$ by a slightly larger number, it is possible to remove the smallness assumption on $c$, at the price of adding a possibly large constant $\nu$ to $A+B$. We state this observation as 
Corollary 2. Let the assumptions of Theorem 1 be satisfied, in particular let $A$ and $B$ satisfy the commutator condition (2.6), however, without any restriction of the size of $c>0$.

Then $A+B$ with domain $D(A) \cap D(B)$ is closed, and there is a number $\nu_{0} \geq 0$ such that $\nu+A+B$ is invertible for every $\nu>\nu_{0}$, and there is a constant $C>0$ such that

$$
\left|(\nu+A+B)^{-1}\right| \leq C / \nu \quad \text { for all } \nu>\nu_{0} .
$$

In particular, the operator $\nu_{0}+A+B$ is sectorial.

Some remarks concerning the commutator condition (2.6) seem to be in order. The natural expression to be considered should be the commutator of the resolvents of $A$ and $B$, i.e.

$$
C(\lambda, \mu):=(\lambda+A)^{-1}(\mu+B)^{-1}-(\mu+B)^{-1}(\lambda+A)^{-1} .
$$

If $A$ and $B$ are subject to the estimates (2.5) and (2.4), then

$$
|C(\lambda, \mu)| \leq \frac{M_{A} M_{B}}{(1+|\lambda|)|\mu|}, \quad \text { for all } \lambda \in \Sigma_{\pi-\varphi_{A}}, \mu \in \Sigma_{\pi-\varphi_{B}} .
$$

This estimate suggests that, instead of (2.6), we look at conditions of the form

$$
|C(\lambda, \mu)| \leq \frac{M_{A} M_{B}}{\left(1+|\lambda|^{p}\right)|\mu|^{q}}, \quad \text { for all } \lambda \in \Sigma_{\pi-\varphi_{A}}, \mu \in \Sigma_{\pi-\varphi_{B}},
$$

where $p$ and $q$ are nonnegative numbers such that $p+q>2$. So far it is not known whether a condition of this type is sufficient to prove noncommutative versions of the Da Prato-Grisvard or Dore-Venni theorems. Observe that

$$
C(\lambda, \mu)=A(\lambda+A)^{-1}\left[A^{-1}(\mu+B)^{-1}-(\mu+B)^{-1} A^{-1}\right] A(\lambda+A)^{-1}
$$

hence (2.6) implies an estimate for $C(\lambda, \mu)$ of the above mentioned type, where $p=1-\alpha$ and $q=1+\beta$, in particular $p+q=2+\beta-\alpha>2$.

\section{Applications to Evolution and Evolutionary Integral Equations}

Let $Y$ be a Banach space of class $\mathcal{H} \mathcal{T}$, let $\{L(t)\}_{t \geq 0}$ be a family of closed linear densely defined operators in $X$, probably with variable domains $D(L(t))$, and $a \in$ $L_{l o c}^{1}\left(\mathbb{R}_{+}\right)$a nontrivial scalar kernel of subexponential growth. The latter means that

$$
\int_{0}^{\infty}|a(t)| e^{-\varepsilon t} d t<\infty \quad \text { for each } \varepsilon>0 .
$$

Consider the following evolutionary integral equation:

$$
u(t)+\int_{0}^{t} a(t-s)[\nu u(s)+L(s) u(s)-f(s)] d s=0, \quad t \geq 0,
$$

where $f: \mathbb{R}_{+} \rightarrow X$ is a given function, strongly measurable and locally integrable, at least. Observe that evolution equations of the type

$$
\dot{u}(t)+L(t) u(t)+\nu u(t)=f(t), \quad t \geq 0, \quad u(0)=0,
$$

are special cases of $(3.1)$; choose $a(t)=1$ and differentiate (3.1) to see this. 
We want to study (3.1) in an $L^{p}$-setting by means of the results from Section 2 . For this purpose let $X=L^{p}\left(\mathbb{R}_{+} ; Y\right)$, where $p \in(1, \infty)$, with norm $|\cdot|_{p}$, and define an operator $A$ in the standard way by means of

$$
\left\{\begin{array}{l}
(A u)(t)=L(t) u(t), \quad \text { for a.a. } t \geq 0, \\
D(A)=\{u \in X: u(t) \in D(L(t)) \text { for a.a. } t \geq 0, A u \in X\} .
\end{array}\right.
$$

Then $A$ is a closed linear operator in $X$. To obtain $A \in B I P(X)$ we impose the following condition on $L(t)$.

(L) For each $t \geq 0, L(t) \in B I P(Y)$, and there are constants $M_{A}, K_{A}>0$ and $\varphi_{A} \in(0, \pi)$ such that

$$
\left|(\lambda+L(t))^{-1}\right| \leq \frac{M_{A}}{1+|\lambda|} \quad \text { for all } t \geq 0, \lambda \in \Sigma_{\pi-\varphi_{A}},
$$

and

$$
\left|L(t)^{i s}\right| \leq K_{A} e^{|s| \varphi_{A}} \quad \text { for all } t \geq 0, s \in \mathbb{R} .
$$

It is easily seen that

$$
\left((\lambda+A)^{-1} f\right)(t)=(\lambda+L(t))^{-1} f(t) \quad \text { for a.a. } t \geq 0 \text { and all } \lambda \in \Sigma_{\pi-\varphi_{A}},
$$

and that

$$
\left(A^{i s} f\right)(t)=L(t)^{i s} f(t) \quad \text { for a.a. } t \geq 0 \text { and all } s \in \mathbb{R} ;
$$

hence $A \in B I P(X)$ and estimates (2.3) and (2.5) are valid for $A$. Observe that $A$ is densely defined, since for a given $f \in X$ the functions $f_{n}=n(n+A)^{-1} f$ belong to $D(A)$, are bounded a.e. by the function $M_{A}|f(t)|$ which belongs to $L^{p}\left(\mathbb{R}_{+}\right)$, and converge to $f(t)$ a.e. in $Y$, hence also in $X$, by Lebesgue's theorem.

The construction of $B$ is not so obvious, except for the case of the evolution equation (3.2), i.e. $a(t)=1$. In fact, in this case

$$
(B u)(t)=\frac{d}{d t} u(t), \quad t \geq 0, \quad D(B)=H_{0}^{1, p}\left(\mathbb{R}_{+} ; Y\right),
$$

where $H_{0}^{1, p}\left(\mathbb{R}_{+} ; Y\right)$ denotes the space of all functions $u: \mathbb{R}_{+} \rightarrow Y$ which are locally absolutely continuous, differentiable a.e., and such that $\dot{u} \in L^{p}\left(\mathbb{R}_{+} ; Y\right)$ and $u(0)=$ 0 . Obviously, $B$ is a closed linear densely defined operator in $X$, and it is easy to compute the resolvent of $B$ :

$$
\left((\mu+B)^{-1} f\right)(t)=\int_{0}^{t} e^{-\mu(t-s)} f(s) d s, \quad t \geq 0,
$$

whenever $\operatorname{Re} \mu>0$. In particular, $B$ is sectorial with spectral angle $\phi_{B}=\pi / 2$. The vector-valued Marcinkiewicz multiplier theorem (cf. Zimmermann [22], Prüss [16]) then implies $B \in B I P(X)$ and $\theta_{B}=\pi / 2$ for the power angle of $B$. Thus assumptions (2.3) for $B$ and (2.4) are valid, provided $\varphi_{B}$ is chosen larger than $\pi / 2$.

For the case of more general kernels $a(t)$ we refer to Theorem 8.6 and Proposition 8.2 of the second author's monograph [16]. The basic assumptions of these results which are valid in spaces $L^{p}\left(\mathbb{R}_{+} ; Y\right)$, where $Y$ belongs to the class $\mathcal{H} \mathcal{T}$, are the following:

(a) $|\arg \widehat{a}(\lambda)| \leq \vartheta_{B}$ and $\left|\lambda \widehat{a}^{\prime}(\lambda) / \widehat{a}(\lambda)\right| \leq \kappa$, for all $\lambda \in \Sigma_{\pi / 2}$. 
Here $\vartheta_{B} \in(0, \pi)$ and $\kappa>0$ are constants, and the hat indicates Laplace transform. Following the terminology in Prüss [16], kernels satisfying an estimate of the form $|\arg \widehat{a}(\lambda)| \leq \vartheta$ on $\Sigma_{\pi / 2}$ will be called $\vartheta$-sectorial, while kernels which are subject to $\left|\lambda^{n} \widehat{a}^{(n)}(\lambda) / \widehat{a}(\lambda)\right| \leq \kappa$ on $\Sigma_{\pi / 2}$ for all $n \leq k$ will be termed $k$-regular. Then the operator $B$ defined formally in terms of Laplace transforms according to

$$
(B u) \hat{(\lambda)}=\frac{\widehat{u}(\lambda)}{\widehat{a}(\lambda)}, \quad \lambda \in \Sigma_{\pi / 2},
$$

gives rise to a closed linear densely defined operator $B$ in $X$, which is sectorial and admits bounded imaginary powers, and satisfies $\phi_{B} \leq \theta_{B} \leq \vartheta_{B}$. In particular, assumptions (2.3) and (2.4) are valid for any $\varphi_{B}>\vartheta_{B}$. The resolvent of $B$ is given by

$$
\left((\mu+B)^{-1} f\right)(t)=\int_{0}^{t} r_{\mu}(t-s) f(s) d s, \quad t \geq 0,
$$

where $r_{\mu}$ denotes the solution of the scalar Volterra equation

$$
r_{\mu}(t)+\mu \int_{0}^{t} a(t-s) r_{\mu}(s) d s=a(t), \quad t \geq 0 .
$$

In particular, for $a(t)=1$, i.e. for the case of evolution equations, we have $r_{\mu}(t)=$ $e^{-\mu t}$, in accordance with (3.3).

Concerning the domain of $B$, we note the following proposition, which is implied by Corollaries 8.1 and 8.2 of Prüss [16] by restriction to the halfline.

Proposition 1. Suppose the kernel $a(t)$ is subject to condition (a). Let $B$ be defined as above and $\rho>0$. Then

(i) $\lim \sup _{r \rightarrow \infty}|\widehat{a}(r)| r^{\rho}<\infty$ implies $D(B) \hookrightarrow H_{0}^{\rho, p}\left(\mathbb{R}_{+} ; Y\right)$;

(ii) $\liminf _{r \rightarrow \infty}|\widehat{a}(r)| r^{\rho}>0$ and $\liminf _{r \rightarrow 0+}|\widehat{a}(r)|>0$ imply $H_{0}^{\rho, p}\left(\mathbb{R}_{+} ; Y\right) \hookrightarrow$ $D(B)$;

(iii) if $\lim _{t \rightarrow 0+} t^{-\rho} \int_{0}^{t} a(s) d s \neq 0, \infty$ exists and in addition $\liminf _{r \rightarrow 0+}|\widehat{a}(r)|>0$, then $D(B)=H_{0}^{\rho, p}\left(\mathbb{R}_{+} ; Y\right)$.

Here the spaces $H_{0}^{\rho, p}\left(\mathbb{R}_{+} ; Y\right)$ are defined as follows: $u \in H_{0}^{\rho, p}\left(\mathbb{R}_{+} ; Y\right)$ if and only if its extension by 0 to all of $\mathbb{R}$ belongs to $H^{\rho, p}(\mathbb{R} ; Y)$; see Prüss [16] for the latter. In particular the traces at $t=0$ of the derivatives of $u \in H_{0}^{\rho, p}\left(\mathbb{R}_{+} ; Y\right)$ which exist are zero. Recall that $\rho>\sigma+1 / p$ implies $H^{\rho, p}(\mathbb{R} ; Y) \hookrightarrow C^{\sigma}(\mathbb{R} ; Y)$.

Observe that the second condition in (ii) and (iii) holds if $a(t)$ is nonnegative and nontrivial, as will be the case in all examples to be considered here. Let us show that the first part of (ii) in Proposition 1 is satisfied with $\rho_{0}=2 \vartheta_{B} / \pi$, whenever the kernel $a(t)$ is subject to (a). For this purpose we take the analytic completion of the Poisson formula for the harmonic function $h(\lambda)=\arg \widehat{a}(\lambda)$, which reads

$$
\log \widehat{a}(\lambda)=\kappa_{0}+\frac{i}{\pi} \int_{-\infty}^{\infty}\left[\frac{1-i \rho \lambda}{\lambda-i \rho}\right] h(i \rho) \frac{d \rho}{1+\rho^{2}},
$$

where $\kappa_{0} \in \mathbb{R}$ is a suitable constant. Considering only real $\lambda>1$ and estimating the real part of this formula, we obtain

$$
|\operatorname{Re} \log \widehat{a}(\lambda)| \leq \kappa_{0}+\rho_{0} \frac{\lambda^{2}+1}{\lambda^{2}-1} \log \lambda \leq \kappa+\rho_{0} \log \lambda,
$$


for some constant $\kappa$. But this implies

$$
|\widehat{a}(\lambda)|=e^{\log |\widehat{a}(\lambda)|} \geq e^{-|\operatorname{Re} \log \widehat{a}(\lambda)|} \geq e^{-\kappa-\rho_{0} \log \lambda}=c \lambda^{-\rho_{0}},
$$

where $c=e^{-\kappa}>0$. Thus as a result we have the inequality

$$
|\widehat{a}(r)| \geq c r^{-\rho_{0}}, \quad \text { for all } r>1 .
$$

Observe that $\rho_{0}<2$ by the sector condition (a).

Returning to the abstract treatment of (3.1), with the definitions of $A$ and $B$ given above, (3.1) can be rewritten in the form as $\nu u+A u+B u=f$, and we are in position to apply Theorem 1 as well as Corollaries 1 and 2, once we have verified the commutator condition (2.6).

Let

$$
Z(\lambda, \mu)=A(\lambda+A)^{-1}\left[A^{-1}(\mu+B)^{-1}-(\mu+B)^{-1} A^{-1}\right]
$$

with the representations of the resolvents of $A$ and $B$ described above we then have

$$
(Z(\lambda, \mu) f)(t)=\int_{0}^{t} r_{\mu}(t-s) L(t)(\lambda+L(t))^{-1}\left[L(t)^{-1}-L(s)^{-1}\right] f(s) d s, \quad t \geq 0 .
$$

Therefore,

$$
|(Z(\lambda, \mu) f)(t)| \leq \int_{0}^{t}\left|r_{\mu}(t-s)\right|\left|L(t)(\lambda+L(t))^{-1}\left[L(t)^{-1}-L(s)^{-1}\right]\right||f(s)| d s, t \geq 0,
$$

and it appears that in order to establish (2.6) we need assumptions on the quantity $\left|L(t)(\lambda+L(t))^{-1}\left[L(t)^{-1}-L(s)^{-1}\right]\right|$ and on the kernels $\left|r_{\mu}(t)\right|$. Let the following conditions be satisfied in addition to (L) and (a).

(C) There exist constants $\alpha \in[0,1), \delta \in(0,1]$ and $M_{1}>0$ such that

$$
\left|L(t)(\lambda+L(t))^{-1}\left[L(t)^{-1}-L(s)^{-1}\right]\right| \leq \frac{M_{1}|t-s|^{\delta}}{1+|\lambda|^{1-\alpha}} \quad \text { for all } t, s \geq 0, \lambda \in \Sigma_{\pi-\varphi_{A}} .
$$

(r) There exist constants $\beta>0$ and $M_{2}>0$ such that

$$
\left|t^{\delta} r_{\mu}\right|_{1} \leq \frac{M_{2}}{|\mu|^{1+\beta}}, \quad \mu \in \Sigma_{\pi-\varphi_{B}} .
$$

Then we obtain the estimates

$$
\begin{aligned}
|Z(\lambda, \mu) f|_{p} & \leq \frac{M_{1}}{1+|\lambda|^{1-\alpha}}\left|\int_{0}^{t}\right| r_{\mu}(t-s)|| t-\left.\left.s\right|^{\delta}|f(s)| d s\right|_{p} \\
& \leq \frac{M_{1}}{1+|\lambda|^{1-\alpha}}\left|t^{\delta} r_{\mu}\right|_{1}|f|_{p} \\
& \leq \frac{M_{1} M_{2}}{\left(1+|\lambda|^{1-\alpha}\right)|\mu|^{1+\beta}}|f|_{p}, \quad \text { for all } \mu \in \Sigma_{\pi-\varphi_{B}}, \lambda \in \Sigma_{\pi-\varphi_{A}},
\end{aligned}
$$

and for each $f \in L^{p}\left(\mathbb{R}_{+} ; Y\right)$. Therefore the commutator condition (2.6) follows, provided $\beta>\alpha$.

In Section 5 we discuss the assumptions (a) and (r) on the kernel $a(t)$ in detail. For the moment we observe that for the case of evolution equations $a(t)=1$ we have $r_{\mu}(t)=e^{-\mu t}$; hence

$$
\left|t^{\delta} r_{\mu}\right|_{1} \leq \Gamma(1+\delta) /(\operatorname{Re} \mu)^{1+\delta}, \quad \mu \in \Sigma_{\pi / 2}, \delta>-1,
$$

where $\Gamma$ means the gamma function. Therefore $\beta=\delta$ in this case, and (2.6) is valid if (C) holds with $0 \leq \alpha<\delta \leq 1$. 
Conditions (L) and (C) on the family of operators $\{L(t)\}_{t \geq 0}$ will be discussed to some extent for the case of elliptic partial differential operators on $Y=L^{q}(\Omega)$ in Section 6. They have been used before for the case of evolution equations by Acquistapace and Terreni [2]. Observe that in the case of constant domains $D(L(t))=D_{0}$ for $t \geq 0$, condition (C) with $\alpha=0$ is implied by the resolvent estimate in (L) and by the classical condition

(C0) There exist constants $\delta \in(0,1]$ and $M_{3}>0$ such that

$$
\left|[L(t)-L(s)] L^{-1}(s)\right| \leq M_{3}|t-s|^{\delta} \quad \text { for all } t, s \geq 0,
$$

which was introduced by Sobolevskii [19] and by Kato and Tanabe (see [20]).

Let us summarize our considerations in

Theorem 2. Let $Y$ be a Banach space of class $\mathcal{H} \mathcal{T},\{L(t)\}_{t \geq 0}$ a family of closed linear densely defined operators in $Y$ which is subject to $(\boldsymbol{L})$ and $(\boldsymbol{C})$, let $a \in$ $L_{\text {loc }}^{1}\left(\mathbb{R}_{+}\right)$be a kernel of subexponential growth which satisfies $(\boldsymbol{a})$ and $(\boldsymbol{r})$, and let $\nu \geq 0$ and $p \in(1, \infty)$. Assume that (3.1) is parabolic in the sense that $\varphi_{A}+\varphi_{B}<\pi$, and assume $\beta>\alpha$.

If either $\nu \geq 0$ is sufficiently large or $M_{1} M_{2}$ is sufficiently small, then for every $f \in L^{p}\left(\mathbb{R}_{+} ; Y\right)$, (3.1) admits a unique solution $u \in L^{p}\left(\mathbb{R}_{+} ; Y\right)$ such that $u(t) \in$ $D(L(t))$ for a.a. $t \geq 0$ and $L(\cdot) u \in L^{p}\left(\mathbb{R}_{+} ; Y\right)$. Moreover, if $\lim _{\sup } \rightarrow \infty|\widehat{a}(r)| r^{\rho}<$ $\infty$ for some $\rho \geq 0$, then $u \in H_{0}^{\rho, p}\left(\mathbb{R}_{+} ; Y\right)$.

In case (3.1) is considered on a finite interval $J=[0, T]$, then no restrictions on the size of $M_{1} M_{2}$ or the magnitude of $\nu$ are needed. In fact, multiplying (3.1) by $e^{-\omega t}$ and setting $v(t)=e^{-\omega t} u(t)$ and $g(t)=e^{-\omega t} f(t),(3.1)$ is transformed into an equation of the same type, with $a(t)$ replaced by $a_{\omega}(t)=e^{-\omega t} a(t)$. This way conditions (a) and (r) remain valid with the same constants. Taking the inverse convolution of this new equation with $\delta_{0}+\left(\nu-\nu_{0}\right) a_{\omega}$, where $\delta_{0}$ denotes the Dirac distribution and $\nu_{0}>0$ is large, there results an equation of the form (3.1) with $\nu$ replaced by $\nu_{0}$ and $a_{\omega}$ by $r_{\omega, \nu-\nu_{0}}$. Then given $\nu_{0}>0$, choosing $\omega$ sufficiently large, (a) and (r) are still satisfied, probably with slightly larger constants. Thus as a corollary to Theorem 2 we obtain

Corollary 3. Assume that the assumptions of Theorem 2 are satisfied, without restrictions on the magnitudes of $\nu \in \mathbb{R}$ or $M_{1} M_{2}$, and let $J=[0, T]$.

Then for every $f \in L^{p}(J ; Y)$, (3.1) admits a unique solution $u \in L^{p}(J ; Y)$ such that $u(t) \in D(L(t))$ for a.a. $t \in J$ and $L(\cdot) u \in L^{p}(J ; Y)$. Moreover, if $\lim \sup _{r \rightarrow \infty}|\widehat{a}(r)| r^{\rho}<\infty$ for some $\rho \geq 0$, then $u \in H_{0}^{\rho, p}(J ; Y)$.

\section{Proof of the Main Results}

In this section, we want to give the proof of Theorem 1 stated in Section 2. For this purpose fix $A, B, \theta_{A}, \theta_{B}, \varphi_{A}, \varphi_{B}, M_{A}, M_{B}, c, K_{A}, K_{B}$ as in Section 2. We begin with a general lemma.

Lemma 1. Let $\left\{F_{t}\right\}_{t \in \mathbb{R}}$ be a strongly measurable family of bounded linear operators in $X$ which is exponentially bounded in the sense that there are constants $K>0$ and $\theta<\pi$ such that

$$
\left|F_{t} x\right| \leq K e^{\theta|t|}|x|, \quad \text { for all } x \in X, t \in \mathbb{R} .
$$


Then, for each $x \in X$ and $a>0$,

the function

$$
\lim _{\varepsilon \rightarrow 0^{+}} \int_{|s| \geq \varepsilon} F_{t-s} x \frac{d s}{\sinh \pi s} \text { exists a.e. on }(-a, a),
$$

$$
\mathcal{F} x: t \mapsto \lim _{\varepsilon \rightarrow 0} \frac{1}{2 i} \int_{|s| \geq \varepsilon} F_{t-s} x \frac{d s}{\sinh \pi s}
$$

belongs to $L^{2}((-a, a) ; X)$ and

$$
|\mathcal{F} x|_{L^{2}((-a, a) ; X)} \leq c\left(a, K, \theta, \mathcal{H}_{2}\right)|x|,
$$

where $c\left(a, K, \theta, \mathcal{H}_{2}\right)$ denotes a constant which only depends on $a, K, \theta$, and the norm $\mathcal{H}_{2}$ of the Hilbert transform in $L^{2}(\mathbb{R}, X)$.

Proof. The proof is based on the boundedness of the Hilbert transform in $L^{2}(\mathbb{R}, X)$, since $X$ is of class $\mathcal{H} \mathcal{T}$. It is basically due to Dore-Venni [9]. For each $\varepsilon \in(0, a)$, we have

$$
\begin{array}{r}
\frac{1}{2 i} \int_{|s| \geq \varepsilon} F_{t-s} x \frac{d s}{\sinh \pi s}=\frac{1}{2 i} \int_{|s| \geq 2 a} F_{t-s} x \frac{d s}{\sinh \pi s} \\
+\frac{1}{2 i} \int_{\varepsilon \leq|s| \leq 2 a} F_{t-s} x\left(\frac{1}{\sinh \pi s}-\frac{1}{\pi s}\right) d s+\frac{1}{2 i \pi} \int_{\varepsilon \leq|s| \leq 2 a} F_{t-s} x \frac{d s}{s} .
\end{array}
$$

It is easy to deal with the first two terms since $\frac{1}{|\sinh \pi s|} \leq 4 e^{-\pi|s|}$ for all $s \in \mathbb{R},|s| \geq$ 1 , and $\left|\frac{1}{\sinh \pi s}-\frac{1}{\pi s}\right|=\frac{\pi|s|}{6}+o(|s|)$ for $|s|$ near 0 . The difficult term is the third one:

$$
\begin{aligned}
\frac{1}{2 i \pi} \int_{\varepsilon \leq|s| \leq 2 a} F_{t-s} x \frac{d s}{s}= & \frac{1}{2 i}\left(H_{\varepsilon} f\right)(t)+\frac{1}{2 i \pi} \int_{-2 a}^{t-2 a} F_{t-s} x \frac{d s}{s} \\
& -\frac{1}{2 i \pi} \int_{2 a}^{t+2 a} F_{t-s} x \frac{d s}{s},
\end{aligned}
$$

where $f(r)=\chi_{(-2 a, 2 a)} F_{r} x$, and $\left(H_{\varepsilon} g\right)(t)=\frac{1}{\pi} \int_{|s| \geq \varepsilon} g(t-s) \frac{d s}{s}$ denotes the truncated Hilbert transform. Since $f \in L^{2}(\mathbb{R}, X)$, we know that $\left(H_{\varepsilon} f\right)(t)$ converges for a.e. $t \in \mathbb{R}$, and in $L^{2}(\mathbb{R}, X)$ as $\varepsilon \rightarrow 0^{+}$. We restrict ourselves to the interval $(-a, a)$ to assure the convergence of the two other terms. The bound of $|\mathcal{F} x|_{\left.L^{2}(-a, a) ; X\right)}$ is now immediate, since $|f|_{L^{2}(\mathbb{R}, X)} \leq 2 K e^{2 \theta a}|x|$.

The next main idea is to approximate $A$ and $B$ by bounded invertible operators. Since $A$ is already invertible, it is sufficient to replace it by $A_{\delta}=A(1+\delta A)^{-1}$, $\delta \in(0,1) ; B$ is approximated by $B_{\delta}=(B+\delta)(1+\delta B)^{-1}, \delta \in(0,1)$. For all $\delta \in(0,1), A_{\delta}$ and $B_{\delta}$ are bounded and invertible operators, and we have

$$
\lim _{\delta \rightarrow 0^{+}} A_{\delta} x=A x \text { for all } x \in D(A) \quad \text { and } \quad \lim _{\delta \rightarrow 0^{+}} B_{\delta} x=B x \text { for all } x \in D(B) .
$$

Moreover, we know that $A_{\delta}$ and $B_{\delta}$ satisfy (2.3) with constants $K_{A}^{\prime}$ and $K_{B}^{\prime}$ independent of $\delta \in(0,1)$; to see this use $A \in B I P(X) \Longleftrightarrow A^{-1} \in B I P(X)$, and apply Theorem 3 of Prüss-Sohr [17]. The operator $B_{\delta}$ also satisfies (2.4) with $M_{B}^{\prime}$ independent of $\delta \in(0,1)$, and for $A_{\delta}$ inequality $(2.5)$ with $M_{A}^{\prime}$ independent of $\delta \in(0,1)$ is valid.

Let us define the following bounded linear operators $S_{\delta}$ and $T_{\delta}$ for each $\delta \in(0,1)$ :

$$
S_{\delta}=\frac{1}{2 i} \int_{\gamma-i \infty}^{\gamma+i \infty} A_{\delta}^{-z} B_{\delta}^{z-1} \frac{d z}{\sin (\pi z)} \quad \text { and } \quad T_{\delta}=\frac{1}{2 i} \int_{\gamma-i \infty}^{\gamma+i \infty} B_{\delta}^{-z} A_{\delta}^{z-1} \frac{d z}{\sin (\pi z)},
$$


where $\gamma \in(0,1)$ is arbitrary; observe that the integrals are absolutely convergent, thanks to (2.3). Since the functions $z \mapsto A_{\delta}^{-z} B_{\delta}^{z}$ and $z \mapsto B_{\delta}^{-z} A_{\delta}^{z}$ are holomorphic on $\mathbb{C}$, the theorem of residues implies the identities

$$
A_{\delta} S_{\delta} x+S_{\delta} B_{\delta} x=x \quad \text { and } \quad T_{\delta} A_{\delta} x+B_{\delta} T_{\delta} x=x, \quad \text { for all } x \in X, \delta \in(0,1) .
$$

We show next that $A_{\delta}$ and $B_{\delta}$ satisfy the commutator condition (2.6) with a constant $c^{\prime}$ independent of $\delta \in(0,1)$.

Lemma 2. Let $Z_{\delta}(\lambda, \mu)=A_{\delta}\left(\lambda+A_{\delta}\right)^{-1}\left[A_{\delta}^{-1}\left(\mu+B_{\delta}\right)^{-1}-\left(\mu+B_{\delta}\right)^{-1} A_{\delta}^{-1}\right]$ for all $\delta \in(0,1)$. Then there exists a constant $c\left(\varphi_{A}, \varphi_{B}\right)$ which depends only on $\varphi_{A}$ and $\varphi_{B}$ such that

$$
\left|Z_{\delta}(\lambda, \mu)\right| \leq \frac{c \cdot c\left(\varphi_{A}, \varphi_{B}\right)}{\left(1+|\lambda|^{1-\alpha}\right)|\mu|^{1+\beta}}, \quad \lambda \in \Sigma_{\pi-\varphi_{A}}, \mu \in \Sigma_{\pi-\varphi_{B}},
$$

where $\alpha$ and $\beta$ denote the same constants as in (2.6).

Proof. A simple calculation gives for all $\delta \in(0,1)$

$$
Z_{\delta}(\lambda, \mu)=\frac{1-\delta^{2}}{(1+\delta \lambda)(1+\delta \mu)^{2}} Z\left(\frac{\lambda}{1+\delta \lambda}, \frac{\mu+\delta}{1+\delta \mu}\right),
$$

for all $\lambda \in \Sigma_{\pi-\varphi_{A}}$ and $\mu \in \Sigma_{\pi-\varphi_{B}}$, where

$$
Z(\lambda, \mu)=A(\lambda+A)^{-1}\left[A^{-1}(\mu+B)^{-1}-(\mu+B)^{-1} A^{-1}\right] .
$$

Then the commutator condition (2.6) gives the expected bound, where

$$
\begin{aligned}
c\left(\varphi_{A}, \varphi_{B}\right)= & \sup \left\{\frac{1}{|1+\delta \lambda|^{\alpha}|1+\delta \mu|^{1-\beta}} ; \lambda \in \Sigma_{\pi-\varphi_{A}}, \mu \in \Sigma_{\pi-\varphi_{B}}, \delta \in(0,1)\right\} \\
& \cdot \sup \left\{\left|\frac{\mu}{\mu+\delta}\right|^{1+\beta} ; \mu \in \Sigma_{\pi-\varphi_{B}}, \delta \in(0,1)\right\} \\
& \cdot \sup \left\{|1+\delta \lambda|^{\alpha-1} ; \lambda \in \Sigma_{\pi-\varphi_{A}}, \delta \in(0,1)\right\} .
\end{aligned}
$$

Let us derive different representations of $S_{\delta}$ and $T_{\delta}, \delta \in(0,1)$. For this purpose we fix $\theta<\varphi_{B}, \varphi<\varphi_{A}$ and $\delta_{0} \in(0,1)$ such that

$$
\left(\bigcup_{0<\delta<\delta_{0}} \sigma\left(B_{\delta}\right) \cup \sigma(B)\right) \subset \Sigma_{\theta} \quad \text { and } \quad\left(\bigcup_{0<\delta<\delta_{0}} \sigma\left(A_{\delta}\right) \cup \sigma(A)\right) \subset \Sigma_{\varphi},
$$

and $\delta \in\left(0, \delta_{0}\right)$. Let $R_{\delta}>\sup \left\{|\mu| ; \mu \in \sigma\left(B_{\delta}\right)\right\}$ and $0<r_{\delta}<\inf \left\{|\mu| ; \mu \in \sigma\left(B_{\delta}\right)\right\}$. We denote by $\Gamma_{B}^{\delta}$ the following contour:

$$
\left[r_{\delta}, R_{\delta}\right] e^{-i \theta} \cup R_{\delta} e^{i[-\theta, \theta]} \cup\left[R_{\delta}, r_{\delta}\right] e^{i \theta} \cup r_{\delta} e^{i[\theta,-\theta]} ;
$$

$\Gamma_{B}^{\delta}$ is a positively directed contour which surrounds $\sigma\left(B_{\delta}\right)$. The functional calculus of Dunford yields

$$
B_{\delta}^{z-1}=\frac{1}{2 i \pi} \int_{\Gamma_{B}^{\delta}} \mu^{z-1}\left(\mu-B_{\delta}\right)^{-1} d \mu \quad \text { for all } z \in \mathbb{C} .
$$

The same argument can be applied to $A_{\delta}$ :

$$
\Gamma_{A}^{\delta}=\left[r_{\delta}^{\prime}, R_{\delta}^{\prime}\right] e^{-i \varphi} \cup R_{\delta}^{\prime} e^{i[-\varphi, \varphi]} \cup\left[R_{\delta}^{\prime}, r_{\delta}^{\prime}\right] e^{i \varphi} \cup r_{\delta}^{\prime} e^{i[\varphi,-\varphi]},
$$


where $R_{\delta}^{\prime}>\sup \left\{|\lambda| ; \lambda \in \sigma\left(A_{\delta}\right)\right\}$ and $0<r_{\delta}^{\prime}<\inf \left\{|\lambda| ; \lambda \in \sigma\left(A_{\delta}\right)\right\}$, and we obtain

$$
A_{\delta}^{-z}=\frac{1}{2 i \pi} \int_{\Gamma_{A}^{\delta}} \lambda^{-z}\left(\lambda-A_{\delta}\right)^{-1} d \lambda \quad \text { for all } z \in \mathbb{C} .
$$

Hence, once $\gamma \in(0,1)$ has been chosen, Fubini's theorem yields

$$
S_{\delta}=\frac{1}{(2 i \pi)^{2}} \int_{\Gamma_{B}^{\delta}} \int_{\Gamma_{A}^{\delta}}\left(\frac{1}{2 i} \int_{\gamma-i \infty}^{\gamma+i \infty} \lambda^{-z} \mu^{z-1} \frac{d z}{\sin \pi z}\right)\left(\lambda-A_{\delta}\right)^{-1}\left(\mu-B_{\delta}\right)^{-1} d \lambda d \mu .
$$

Since $|\arg \lambda|+|\arg \mu| \leq \varphi+\theta<\pi$, the inverse Mellin transform gives

$$
\frac{1}{\lambda+\mu}=\frac{1}{2 i} \int_{\gamma-i \infty}^{\gamma+i \infty} \lambda^{-z} \mu^{z-1} \frac{d z}{\sin \pi z}
$$

and the functional calculus of Dunford implies that

$$
\frac{1}{2 i \pi} \int_{\Gamma_{A}^{\delta}} \frac{1}{\lambda+\mu}\left(\lambda-A_{\delta}\right)^{-1} d \lambda=\left(\mu+A_{\delta}\right)^{-1} .
$$

Therefore

$$
S_{\delta}=\frac{1}{2 i \pi} \int_{\Gamma_{B}^{\delta}}\left(\mu+A_{\delta}\right)^{-1}\left(\mu-B_{\delta}\right)^{-1} d \mu .
$$

Thanks to the estimates (2.4) and (2.5), by holomorphy we may deform the contour $\Gamma_{B}^{\delta}$ into $\Gamma=(\infty, 0] e^{i \theta} \cup[0, \infty) e^{-i \theta}$, which leads to

$$
S_{\delta}=\frac{1}{2 i \pi} \int_{\Gamma}\left(\mu+A_{\delta}\right)^{-1}\left(\mu-B_{\delta}\right)^{-1} d \mu, \quad \text { for all } \delta \in\left(0, \delta_{0}\right) .
$$

In the same way, we can show that

$$
T_{\delta}=\frac{1}{2 i \pi} \int_{\Gamma}\left(\mu-B_{\delta}\right)^{-1}\left(\mu+A_{\delta}\right)^{-1} d \mu, \quad \text { for all } \delta \in\left(0, \delta_{0}\right),
$$

with the same contour $\Gamma$.

As $\delta \rightarrow 0^{+}$the integrands in these formulas converge strongly for every $\mu \in \Gamma$, and are uniformly bounded by a function which is integrable on $\Gamma$. Therefore by Lebesgue's theorem

$$
\lim _{\delta \rightarrow 0^{+}} S_{\delta} x=\frac{1}{2 i \pi} \int_{\Gamma}(\mu+A)^{-1}(\mu-B)^{-1} x d \mu=: S x, \quad x \in X,
$$

and

$$
\lim _{\delta \rightarrow 0^{+}} T_{\delta} x=\frac{1}{2 i \pi} \int_{\Gamma}(\mu-B)^{-1}(\mu+A)^{-1} x d \mu=: T x, \quad x \in X .
$$

We are now in position to state the following lemma, which is the main step in the proof of invertibility of $A+B$.

Lemma 3. $S$ maps $X$ into $D(A), A S \in \mathcal{B}(X)$ and $A S x+S B x=x$ for all $x \in$ $D(B)$. Consequently $S B$ admits a unique bounded extension to all of $X$.

Proof. (i) For all $\delta \in\left(0, \delta_{0}\right)$ and for all $x \in D(B), \lim _{\delta \rightarrow 0^{+}} S_{\delta} B_{\delta} x=S B x$ since $S_{\delta} \rightarrow S$ strongly as $\delta \rightarrow 0^{+}$and $B_{\delta} x \rightarrow B x$ as $\delta \rightarrow 0^{+}$for $x \in D(B)$. Then $A_{\delta} S_{\delta} x \rightarrow$ $x-S B x$ as $\delta \rightarrow 0^{+}$, since $A_{\delta} S_{\delta} x+S_{\delta} B_{\delta} x=x$ for all $\delta \in(0,1)$.

On the other hand, $A_{\delta} S_{\delta} x=A(1+\delta A)^{-1} S_{\delta} x$ and $(1+\delta A)^{-1} x \rightarrow x$ for all $x \in X$ as $\delta \rightarrow 0^{+}$; hence $(1+\delta A)^{-1} S_{\delta} x \rightarrow S x$ as $\delta \rightarrow 0^{+}$. Therefore, since $A$ is closed, $S x \in D(A)$ and $A S x=x-S B x$ for all $x \in D(B)$. 
(ii) For all $x \in X$ and all $\delta \in\left(0, \delta_{0}\right)$, we have

$$
S_{\delta} B_{\delta}=\frac{1}{2 i} \int_{\gamma-i \infty}^{\gamma+i \infty} A_{\delta}^{-z} B_{\delta}^{z} \frac{d z}{\sin (\pi z)}, \gamma \in(0,1) .
$$

To show that $S_{\delta} B_{\delta}$ is bounded uniformly w.r.t. $\delta \in(0,1)$, write

$$
A_{\delta}^{-z} B_{\delta}^{z}=A_{\delta}^{-i t}\left(A_{\delta}^{i t-z} B_{\delta}^{z}-B_{\delta}^{z} A_{\delta}^{i t-z}\right)+A_{\delta}^{-i t} B_{\delta}^{i t} B_{\delta}^{z-i t} A_{\delta}^{i t-z}
$$

Since $z \mapsto B_{\delta}^{z} A_{\delta}^{-z} \in \mathcal{B}(X)$ is holomorphic in $\{z \in \mathbb{C} ; 0<\operatorname{Re} z<1\}$ thanks to Lemma 1 with $F_{t}^{\delta} x=B_{\delta}^{-i t} A_{\delta}^{i t} x$, shifting the contour to the imaginary axis, we have for a.a. $t \in\left(-\frac{1}{2}, \frac{1}{2}\right)$

$$
\frac{1}{2 i} \int_{\gamma-i \infty}^{\gamma+i \infty} A_{\delta}^{-i t} B_{\delta}^{i t} B_{\delta}^{z-i t} A_{\delta}^{i t-z} \frac{d z}{\sin (\pi z)}=\frac{1}{2} x+A_{\delta}^{-i t} B_{\delta}^{i t}\left(\mathcal{F}_{\delta} x\right)(t),
$$

where $\mathcal{F}_{\delta} x$ corresponds to $F_{t}^{\delta}$ as in Lemma 1 . On the other hand, thanks to the functional calculus of Dunford, we have

$$
\begin{aligned}
& A_{\delta}^{i t-z} B_{\delta}^{z}-B_{\delta}^{z} A_{\delta}^{i t-z} \\
& =\frac{1}{(2 i \pi)^{2}} \int_{\Gamma_{A}^{\delta}} \int_{\Gamma_{B}^{\delta}} \lambda^{i t-z} \mu^{z}\left[\left(\lambda-A_{\delta}\right)^{-1}\left(\mu-B_{\delta}\right)^{-1}-\left(\mu-B_{\delta}\right)^{-1}\left(\lambda-A_{\delta}\right)^{-1}\right] d \mu d \lambda \\
& \quad=\frac{-1}{(2 i \pi)^{2}} \int_{\Gamma_{A}^{\delta}} \int_{\Gamma_{B}^{\delta}} \lambda^{i t-z} \mu^{z} Z_{\delta}(-\lambda,-\mu) A_{\delta}\left(\lambda-A_{\delta}\right)^{-1} d \mu d \lambda,
\end{aligned}
$$

and therefore integration over $t \in\left[-\frac{1}{2}, \frac{1}{2}\right]$ yields

$$
\begin{aligned}
S_{\delta} B_{\delta} x=\frac{1}{2} x+ & \int_{-\frac{1}{2}}^{\frac{1}{2}} A_{\delta}^{-i t} B_{\delta}^{i t}\left(\mathcal{F}_{\delta} x\right)(t) d t \\
& +\frac{1}{2 i \pi} \int_{\Gamma_{A}^{\delta}}\left(\int_{-\frac{1}{2}}^{\frac{1}{2}} \lambda^{i t} A_{\delta}^{-i t} d t\right) \lambda Z_{\delta}(-\lambda, \lambda) A_{\delta}\left(\lambda-A_{\delta}\right)^{-1} x d \lambda,
\end{aligned}
$$

since

$$
\frac{1}{2 i} \int_{\gamma-i \infty}^{\gamma+i \infty} \lambda^{-z} \mu^{z} \frac{d z}{\sin \pi z}=\frac{\mu}{\mu+\lambda}
$$

by the inverse Mellin transform, for any $\gamma \in(0,1), \lambda \in \Gamma_{A}^{\delta}$ and $\mu \in \Gamma_{B}^{\delta}$, and

$$
\frac{1}{2 i \pi} \int_{\Gamma_{B}^{\delta}} \frac{\mu}{\lambda+\mu}\left(\mu-B_{\delta}\right)^{-1} d \mu=B_{\delta}\left(\lambda+B_{\delta}\right)^{-1}
$$

by the Dunford calculus. As in the derivation of (4.1) we may deform the contour $\Gamma_{A}^{\delta}$ into $\Gamma^{\prime}=(\infty, 0) e^{i \varphi} \cup(0, \infty) e^{-i \varphi}$. Therefore we obtain the following estimate:

$$
\begin{aligned}
& \left|S_{\delta} B_{\delta} x\right| \leq \frac{1}{2}|x|+K_{A}^{\prime} K_{B}^{\prime} e^{\pi / 2}\left|\mathcal{F}_{\delta} x\right|_{L^{2}\left(-\frac{1}{2}, \frac{1}{2} ; X\right)} \\
& +K_{A}^{\prime} \frac{e^{\pi}}{2 \pi}\left|\int_{\Gamma^{\prime}}\right| \lambda|| Z_{\delta}(-\lambda, \lambda)\left|\left(M_{A}^{\prime}+1\right)\right| x|| d \lambda|| \\
& \leq \frac{1}{2}|x|+K_{A}^{\prime} K_{B}^{\prime} e^{\pi / 2} c\left(K_{A}^{\prime} K_{B}^{\prime}, \phi_{A}+\phi_{B}, \mathcal{H}_{2}\right) \\
& +c^{\prime}\left(M_{A}^{\prime}+1\right) K_{A}^{\prime} \frac{e^{\pi}}{\pi}\left(\int_{0}^{\infty} \frac{d r}{\left(1+r^{1+\alpha}\right) r^{\beta}}\right)|x| \\
& \leq C|x|, \quad \text { for all } x \in X,
\end{aligned}
$$


where $C>0$ denotes a constant which is independent of $x \in X$ and $\delta \in\left(0, \delta_{0}\right)$. Since $D(B)$ is dense in $X$ and $\lim _{\delta \rightarrow 0^{+}} S_{\delta} B_{\delta} x=S B x$ for all $x \in D(B), S B$ is bounded and admits a unique bounded extension $\overline{S B}$ on $X$. Since $A$ is closed, $S x \in D(A)$ and $A S x+\overline{S B} x=x$ for all $x \in X$. Moreover, $A S=1-\overline{S B} \in \mathcal{B}(X)$.

We now construct the operator $Q$ as announced in Section 2 .

Lemma 4. Let $Q=A S-A^{2} S A^{-1}$. Then $Q \in \mathcal{B}(X)$ and $|Q|<1$, provided the constant c from (2.6) is small enough.

Proof. Let

$$
\begin{aligned}
Q_{\delta} & =A_{\delta} S_{\delta}-A_{\delta}^{2} S_{\delta} A_{\delta}^{-1} \\
& =\frac{1}{2 i} \int_{\gamma-i \infty}^{\gamma+i \infty} A_{\delta}^{-z+2}\left(A_{\delta}^{-1} B_{\delta}^{z-1}-B_{\delta}^{z-1} A_{\delta}^{-1}\right) \frac{d z}{\sin \pi z},
\end{aligned}
$$

for $\gamma \in(0,1)$ and $\delta \in\left(0, \delta_{0}\right)$. As before we have

$$
B_{\delta}^{z-1}=\frac{1}{2 i \pi} \int_{\Gamma_{B}^{\delta}} \mu^{z-1}\left(\mu-B_{\delta}\right)^{-1} d \mu .
$$

Hence by Fubini's theorem

$$
\begin{aligned}
Q_{\delta}=\frac{1}{2 i \pi} \int_{\Gamma_{B}^{\delta}}\left(\frac{1}{2 i} \int_{\gamma-i \infty}^{\gamma+i \infty} \mu^{z-1} A_{\delta}^{-z+2} \frac{d z}{\sin \pi z}\right) \\
\cdot\left(A_{\delta}^{-1}\left(\mu-B_{\delta}\right)^{-1}-\left(\mu-B_{\delta}\right)^{-1} A_{\delta}^{-1}\right) d \mu .
\end{aligned}
$$

The theorem of residues implies

$$
\left(1+\mu A_{\delta}^{-1}\right) \frac{1}{2 i} \int_{\gamma-i \infty}^{\gamma+i \infty} \mu^{z-1} A_{\delta}^{-z+2} \frac{d z}{\sin \pi z}=-\frac{1}{2 i} 2 i \pi \operatorname{Res}_{z=1}\left(\frac{\mu^{z-1} A_{\delta}^{-z+2}}{\sin \pi z}\right)=A_{\delta} .
$$

Therefore

$$
\begin{aligned}
Q_{\delta} & =\frac{1}{2 i \pi} \int_{\Gamma_{B}^{\delta}} A_{\delta}\left(1+\mu A_{\delta}^{-1}\right)^{-1}\left(A_{\delta}^{-1}\left(\mu-B_{\delta}\right)^{-1}-\left(\mu-B_{\delta}\right)^{-1} A_{\delta}^{-1}\right) d \mu \\
& =\frac{1}{2 i \pi} \int_{\Gamma_{B}^{\delta}} A_{\delta}\left(1-\mu\left(\mu+A_{\delta}\right)^{-1}\right)\left(A_{\delta}^{-1}\left(\mu-B_{\delta}\right)^{-1}-\left(\mu-B_{\delta}\right)^{-1} A_{\delta}^{-1}\right) d \mu \\
& =\frac{1}{2 i \pi} \int_{\Gamma_{B}^{\delta}} \mu Z_{\delta}(\mu,-\mu) d \mu,
\end{aligned}
$$

since $\int_{\Gamma_{B}^{\delta}}\left(\mu-B_{\delta}\right)^{-1} d \mu=1$.

By means of the commutator condition (2.6) and Lebesgue's theorem, deforming first $\Gamma_{B}^{\delta}$ into $\Gamma$ as before, we arrive at

$$
\lim _{\delta \rightarrow 0^{+}} Q_{\delta} x=\frac{1}{2 i \pi} \int_{\Gamma} \mu Z(\mu,-\mu) x d \mu=: Q x, \quad x \in X .
$$

In particular, $Q \in \mathcal{B}(X)$,

$$
\left|Q_{\delta}\right| \leq \frac{c^{\prime}}{\pi} \int_{0}^{\infty} \frac{1}{\left(1+r^{1-\alpha}\right) r^{\beta}} d r<\infty
$$

and

$$
|Q| \leq \frac{c}{\pi} \int_{0}^{\infty} \frac{1}{\left(1+r^{1-\alpha}\right) r^{\beta}} d r .
$$


It is then possible to choose $c_{1}>0$ such that for all $c<c_{1},|Q|$ and $\left|Q_{\delta}\right|$ are smaller than 1.

Finally, $Q_{\delta} x-A_{\delta} S_{\delta} x \longrightarrow Q x-A S x$ and $(1+\delta A)^{-1} A_{\delta} S_{\delta} A_{\delta}^{-1} x \longrightarrow A S A^{-1} x$ as $\delta \rightarrow 0^{+}$, for all $x \in X$, and therefore with $A_{\delta}^{2} S_{\delta} A_{\delta}^{-1} x=A(1+\delta A)^{-1} A_{\delta} S_{\delta} A_{\delta}^{-1} x$, closedness of $A$ implies $A S A^{-1} x \in D(A)$ and $Q x=A S x-A^{2} A A^{-1} x$, for all $x \in X$.

We are now in position to obtain a left inverse of $(A+B, D(A) \cap D(B)): L \in$ $\mathcal{B}(X)$ and $L(A x+B x)=x$ for all $x \in D(A) \cap D(B)$.

Proposition 2. Let $c$ in (2.6) be small enough (as in Lemma 4) and define $L=$ $A^{-1}(1+Q)^{-1} A S$. Then $L \in \mathcal{B}(X)$ and $L(A x+B x)=x$ for all $x \in D(A) \cap D(B)$. The range of $L$ is contained in the domain of $A$.

Proof. Let $L_{\delta}=A_{\delta}^{-1}\left(1+Q_{\delta}\right)^{-1} A_{\delta} S_{\delta}$ for every $\delta \in(0,1)$. We have

$$
L_{\delta}\left(A_{\delta} x+B_{\delta} x\right)=x \quad \text { for all } x \in X,
$$

since $A_{\delta} S_{\delta} x+S_{\delta} B_{\delta} x=x$. Next, $\lim _{\delta \rightarrow 0^{+}} A_{\delta}^{-1}\left(1+Q_{\delta}\right)^{-1} x=A^{-1}(1+Q)^{-1} x$ and the relation $\lim _{\delta \rightarrow 0^{+}} A_{\delta} S_{\delta} x=A S x$, for all $x \in X$, imply $\lim _{\delta \rightarrow 0^{+}} L_{\delta} x=L x$ for all $x \in X$. The operator $L$ is obviously bounded, since $A$ is invertible, $|Q|<1$ and $A S$ is bounded thanks to Lemma 3. Using the relation $L_{\delta} A_{\delta} x+L_{\delta} B_{\delta} x=x$ for all $x \in X$ and all $\delta \in(0,1)$, since

$$
\lim _{\delta \rightarrow 0^{+}} A_{\delta} x=A x \quad \text { and } \quad \lim _{\delta \rightarrow 0^{+}} B_{\delta} x=B x
$$

for all $x \in D(A) \cap D(B)$, we obtain $L(A x+B x)=x$ for all $x \in D(A) \cap D(B)$. Obviously, $R(L) \subset D(A)$, and $A L=(1+Q)^{-1} A S$ is bounded.

We know by now that $L$ is a bounded operator on $X$ and a left inverse of $A+B$ with domain $D(A) \cap D(B)$ and maps $X$ into $D(A)$. We next construct a right inverse $R$ of $(A+B, D(A) \cap D(B))$ using similar methods as for $L$.

Lemma 5. TA defined on $D(A)$ is bounded in $X$ and admits a unique bounded extension $\overline{T A}$ to all of $X$. Moreover, $R(T) \subset D(B)$ and $B T \in \mathcal{B}(X)$.

Proof. Formulas (4.2) and (4.3) imply

$$
\begin{gathered}
A_{\delta} S_{\delta}-T_{\delta} A_{\delta} \\
=\frac{1}{2 i \pi} \int_{\Gamma} \mu A_{\delta}\left(\mu+A_{\delta}\right)^{-1}\left(\left(\mu-B_{\delta}\right)^{-1} A_{\delta}^{-1}-A_{\delta}^{-1}\left(\mu-B_{\delta}\right)^{-1}\right) A_{\delta}\left(\mu+A_{\delta}\right)^{-1} d \mu \\
=\frac{1}{2 i \pi} \int_{\Gamma} \mu Z_{\delta}(\mu,-\mu) A_{\delta}\left(\mu+A_{\delta}\right)^{-1} d \mu
\end{gathered}
$$

Passing to the limit as $\delta \rightarrow 0^{+}$, Lebesgue's theorem yields

$$
A S-T A=\frac{1}{2 i \pi} \int_{\Gamma} \mu Z(\mu,-\mu) A(\mu+A)^{-1} d \mu .
$$

Thanks to the commutator condition (2.6), the right hand side of the last equation defines a bounded operator. Since $A S$ is bounded, we have the expected result. The remaining assertions follow from $B_{\delta} T_{\delta}+T_{\delta} A_{\delta}=1$, which implies with $\delta \rightarrow 0^{+}$ the identity $B T x+T A x=x$, for all $x \in D(A)$. 
Before we construct the operator $P$ of Section 2, observe that by virtue of the moments inequality

$$
\mid A^{\gamma}\left(\lambda+A^{-1} \mid \leq \frac{M}{(1+|\lambda|)^{1-\gamma}} \text { for all } \lambda \in \Sigma_{\phi_{A}}, \gamma \in[0,1],\right.
$$

where $M>0$ denotes a constant independent of $\gamma \in[0,1]$.

Lemma 6. There exists a constant $\gamma \in(0,1)$ such that $P=A^{-\gamma}(T A-A T) A^{\gamma}$ defines a bounded operator and $|P|<1$, whenever the constant $c$ from (2.6) is small enough.

Proof. For every $\delta \in(0,1)$, we have

$$
T_{\delta}=\frac{1}{2 i \pi} \int_{\Gamma_{B}^{\delta}}\left(\mu-B_{\delta}\right)^{-1}\left(\mu+A_{\delta}\right)^{-1} d \mu
$$

Therefore

$$
\begin{aligned}
P_{\delta} & =A_{\delta}^{-\gamma}\left(T_{\delta} A_{\delta}-A_{\delta} T_{\delta}\right) A_{\delta}^{\gamma} \\
& =\frac{1}{(2 i \pi)^{2}} \int_{\Gamma_{A}^{\delta}} \int_{\Gamma_{B}^{\delta}} \lambda^{-\gamma}\left(\lambda-A_{\delta}\right)^{-1}\left(\left(\mu-B_{\delta}\right)^{-1}\left(\mu+A_{\delta}\right)^{-1} A_{\delta}\right. \\
& \left.=\frac{1}{(2 i \pi)^{2}} \int_{\Gamma_{A}^{\delta}} \int_{\Gamma_{B}^{\delta}} \lambda^{-\gamma} Z_{\delta}\left(-\lambda-B_{\delta}\right)^{-1}\left(\mu+A_{\delta}\right)^{-1}\right) A_{\delta}^{\gamma} d \mu d \lambda \\
& \left.=\frac{1}{(2 i \pi)^{2}} \int_{\Gamma^{\prime}} \int_{\Gamma} \lambda^{-\gamma} \mu Z_{\delta}(-\lambda,-\mu) A_{\delta}^{\gamma}\right)^{-1} A_{\delta}^{\gamma} d \mu d \lambda
\end{aligned}
$$

where $\Gamma$ and $\Gamma^{\prime}$ are as before, and since $\int_{\Gamma_{B}^{\delta}}\left(\mu-B_{\delta}\right)^{-1} d \mu=1$. The convergence of both integrals is due to the commutator condition (2.6) and the remark before Lemma 6 , if we choose $\gamma \in(\alpha, \beta)$. Moreover, thanks to Lebesgue's theorem, we have

$$
\lim _{\delta \rightarrow 0^{+}} P_{\delta} x=\frac{1}{(2 i \pi)^{2}} \int_{\Gamma^{\prime}} \int_{\Gamma} \lambda^{-\gamma} \mu Z(-\lambda,-\mu) A^{\gamma}(\mu+A)^{-1} x d \mu d \lambda=: P x, \quad x \in X .
$$

Furthermore, with $r=|\lambda|$ and $s=|\mu|$,

$$
\left|P_{\delta}\right| \leq M_{A}^{\prime} \frac{c^{\prime}}{\pi^{2}} \int_{0}^{\infty} \frac{r^{-\gamma}}{1+r^{1-\alpha}} d r \int_{0}^{\infty} \frac{s^{-\beta}}{(1+s)^{1-\gamma}} d s
$$

and

$$
|P| \leq M_{A} \frac{c}{\pi^{2}} \int_{0}^{\infty} \frac{r^{-\gamma}}{1+r^{1-\alpha}} d r \int_{0}^{\infty} \frac{s^{-\beta}}{(1+s)^{1-\gamma}} d s
$$

It is then possible to choose $c_{2}>0$ such that for all $c \leq c_{2},|P|,\left|P_{\delta}\right|<1$ for all $\delta \in\left(0, \delta_{0}\right)$.

We are now in position to obtain a right inverse of the operator $A+B$ with domain $D(A) \cap D(B)$ : in other words, an operator $R \in \mathcal{B}(X)$, such that $R$ maps $X$ into $D(A) \cap D(B)$ and $(A+B) R x=x$ for all $x \in X$.

Proposition 3. Let the constant $c$ in (2.6) be smaller than $c_{1}$ and $c_{2}$, choose $\gamma \in$ $(\alpha, \beta)$, and define $R=\overline{T A} A^{\gamma-1}(1-P)^{-1} A^{-\gamma}$. Then $R \in \mathcal{B}(X), R$ maps $X$ into $D(A) \cap D(B)$, and $(A+B) R x=x$ for all $x \in X$. Moreover $R=L$, and it is the inverse of $A+B$. 
Proof. Let $R_{\delta}=T_{\delta} A_{\delta}^{\gamma}\left(1-P_{\delta}\right)^{-1} A_{\delta}^{-\gamma}$. Then $\left(A_{\delta}+B_{\delta}\right) R_{\delta} x=x$ for all $x \in X$, since $T_{\delta} A_{\delta}+B_{\delta} T_{\delta}=1$. The operator $R_{\delta}$ is a right inverse of $A_{\delta}+B_{\delta}$; therefore, $R_{\delta}=L_{\delta}$ for every $\delta \in\left(0, \delta_{0}\right)$, since $L_{\delta}$ is the left inverse of $A_{\delta}+B_{\delta} \in \mathcal{B}(X)$. Moreover,

$$
\lim _{\delta \rightarrow 0^{+}} R_{\delta} x=\lim _{\delta \rightarrow 0^{+}} T_{\delta} A_{\delta}^{\gamma}\left(1-P_{\delta}\right)^{-1} A_{\delta}^{-\gamma} x=\overline{T A} A^{\gamma-1}(1-P)^{-1} A^{-\gamma} x=R x, x \in X,
$$

and hence

$$
R x=\lim _{\delta \rightarrow 0^{+}} R_{\delta} x=\lim _{\delta \rightarrow 0^{+}} L_{\delta}\left(A_{\delta}+B_{\delta}\right) R_{\delta}=\lim _{\delta \rightarrow 0^{+}} L_{\delta} x=L x, \text { for all } x \in X .
$$

Thus $R=L$ and $R \in \mathcal{B}(X)$. Since $L$ maps $X$ into $D(A), R$ maps $X$ into $D(A)$ as well. Since $A$ is closed, we have

$$
\lim _{\delta \rightarrow 0^{+}} A_{\delta} R_{\delta} x=A R x \text { for all } x \in X,
$$

and we know moreover that $\left(A_{\delta}+B_{\delta}\right) R_{\delta} x=x$ for all $x \in X$. Since $B$ is closed, this implies $R x \in D(B)$ and $(A+B) R x=x$ for all $x \in X$.

Let $c_{0}=\min \left\{c_{1}, c_{2}\right\}$. The proof of Theorem 1 is now complete.

\section{L1-Estimates for Scalar Resolvent Kernels}

In this section we discuss the assumptions (a) and (r) concerning the kernel $a(t)$ for three special classes of kernels.

1) The first class of kernels consists of the functions

$$
a(t)=t^{\gamma-1} / \Gamma(\gamma), \quad t>0, \text { where } \gamma \in(0,2) .
$$

The special case $\gamma=1$ has been treated already in Section 3 and therefore we shall not pay particular attention to it here. The Laplace transform $\widehat{a}(\lambda)$ of $a(t)$ is then given by

$$
\widehat{a}(\lambda)=\lambda^{-\gamma}, \quad \lambda \in \Sigma_{\pi / 2} .
$$

Thus one obtains $\widehat{a}(\lambda) \neq 0$ and

$$
|\arg \widehat{a}(\lambda)| \leq \gamma \pi / 2<\pi, \quad \lambda \in \Sigma_{\pi / 2},
$$

as well as

$$
-\lambda \widehat{a}^{\prime}(\lambda) / \widehat{a}(\lambda)=\gamma, \quad \lambda \in \Sigma_{\pi / 2} .
$$

This shows that assumption (a) is satisfied with $\vartheta_{B}=\gamma \pi / 2$.

The Laplace transform $\widehat{r}_{\mu}$ of the resolvent kernel $r_{\mu}$ for $\mu>0$ is given by

$$
\widehat{r}_{\mu}(\lambda)=\frac{\widehat{a}(\lambda)}{1+\mu \widehat{a}(\lambda)}=\frac{1}{\mu+\lambda^{\gamma}}, \quad \lambda \in \Sigma_{\pi / 2} .
$$

The dilation property of the Laplace transform then implies

$$
r_{\mu}(t)=|\mu|^{p-1} r_{e^{i \phi}}\left(|\mu|^{p} t\right), \quad t>0
$$

where $\phi=\arg \mu$ and $p=1 / \gamma$. Therefore, we obtain the estimate

$$
\begin{aligned}
\left|t^{\delta} r_{\mu}\right|_{1} & =\int_{0}^{\infty} t^{\delta}\left|r_{\mu}(t)\right| d t=|\mu|^{p-1} \int_{0}^{\infty} t^{\delta}\left|r_{e^{i \phi}}\left(|\mu|^{p} t\right)\right| d t \\
& =|\mu|^{-p \delta-1} \int_{0}^{\infty} t^{\delta}\left|r_{e^{i \phi}}(t)\right| d t=\frac{R(\delta, \phi)}{|\mu|^{1+\delta / \gamma}}
\end{aligned}
$$


where

$$
R(\delta, \phi)=\int_{0}^{\infty} t^{\delta}\left|r_{e^{i \phi}}(t)\right| d t .
$$

Thus to prove (r) for the class of kernels under consideration we need to find bounds for $R(\delta, \phi)$. For this purpose choose any angle $\phi_{0}<\pi-\vartheta_{B}=\pi-\pi \gamma / 2$, and let $\psi>\pi / 2$ be such that $\gamma \psi<\pi-\phi_{0}$. Let $\Gamma$ denote the contour

$$
\begin{aligned}
\Gamma= & (\infty, 1 / 2] e^{-i \psi} \cup\left(e^{-i \psi} / 2, e^{-i \pi} / 2\right) \cup\left[e^{-i \pi} / 2,0\right] \\
& \cup\left[0, e^{i \pi} / 2\right] \cup\left(e^{i \pi} / 2, e^{i \psi} / 2\right) \cup[1 / 2, \infty) e^{i \psi} ;
\end{aligned}
$$

then the function $g(\lambda)=e^{i \phi}+\lambda^{\gamma}$ is holomorphic to the right of $\Gamma$ and all its possible zeros are to the left of this contour, for each $|\phi| \leq \phi_{0}$. Therefore we may deform the integration path in the complex Laplace inversion formula for $r_{e^{i \phi}}$ to $\Gamma$. Since $|g(\lambda)|$ is nonzero and continuous on $\Gamma$ and behaves like $|\lambda|^{\gamma}$ for large $|\lambda|$, by a simple calculation which takes into account the cancellations on the parts of $\Gamma$ which are contained in the negative real half-line, one obtains the following estimate for $r_{e^{i \phi}}$ :

$$
\left|r_{e^{i \phi}}(t)\right| \leq C \int_{0}^{\infty} e^{-r t} \frac{r^{\gamma}}{1+r^{2 \gamma}} d r, \quad \text { for all } t>0,|\phi| \leq \phi_{0},
$$

where $C>0$ denotes a constant which only depends on $\gamma, \phi_{0}$, and $\psi$. But this then implies

$$
R(\delta, \phi) \leq C \int_{0}^{\infty} \int_{0}^{\infty} t^{\delta} e^{-r t} \frac{r^{\gamma}}{1+r^{2 \gamma}} d r d t=C \Gamma(1+\delta) \int_{0}^{\infty} \frac{r^{\gamma-\delta-1}}{1+r^{2 \gamma}} d r<\infty,
$$

for all $|\phi| \leq \phi_{0}$, provided $\delta>-1$ and $|\delta|<\gamma$.

Let us summarize these results as

Proposition 4. Let $\gamma \in(0,2),|\delta|\langle\gamma, \delta\rangle-1$, and consider the kernel $a(t)=$ $t^{\gamma-1} / \Gamma(\gamma), t>0$. Then (a) is satisfied with $\vartheta_{B}=\pi \gamma / 2$, and $(\mathbf{r})$ holds with $\beta=\delta / \gamma$, for any $\varphi_{B}>\vartheta_{B}$.

It is not difficult to see that for $\gamma \neq 1$ the restrictions on $\delta$ in Proposition 4 are essential. In fact, contracting the contour $\Gamma$ from the above proof to the negative real axis, one obtains the representation

$$
r_{1}(t)=\frac{\sin (\pi \gamma)}{\pi} \int_{0}^{\infty} e^{-r t} \frac{r^{\gamma}}{1+2 r^{\gamma} \cos (\pi \gamma)+r^{2 \gamma}} d r
$$

This representation shows that $r_{1}(t)>0$ for all $t>0$, and therefore by Fubini's theorem we have $t^{\delta} r_{1}(t) \in L^{1}\left(\mathbb{R}_{+}\right)$if and only if $|\delta|<\gamma$.

For the case $\gamma \in(1,2)$ the function $g(\lambda)=1+\lambda^{\gamma}$ has zeros at $\lambda=e^{ \pm i \pi / \gamma}$; therefore, contracting the contour $\Gamma$ to the negative real axis, we obtain by the residue theorem

$$
\begin{aligned}
r_{1}(t)=\frac{\sin (\pi \gamma)}{\pi} \int_{0}^{\infty} e^{-r t} \frac{r^{\gamma}}{1+2 r^{\gamma} \cos (\pi \gamma)}+r^{2 \gamma} & d r \\
& -\frac{2}{\gamma} e^{t \cos (\pi / \gamma)} \cos [\pi / \gamma+t \sin (\pi / \gamma)] .
\end{aligned}
$$

In this case $r_{1}(t)$ is no longer nonnegative; however, the second term in this representation has moments of all orders $\delta>-1$ in $L^{1}\left(\mathbb{R}_{+}\right)$, and the first term is negative for all $t>0$. Thus we see that for the case $\gamma \in(1,2), t^{\delta} r_{1} \in L^{1}\left(\mathbb{R}_{+}\right)$if and only if $-1<\delta<\gamma$.

Therefore the estimates in Proposition 4 are optimal. 
2) Next we consider the case where $a(t)$ is a completely positive function, or equivalently $\Phi(\lambda)=1 / \widehat{a}(\lambda)$ is a Bernstein function, which means $\Phi(\lambda)>0$ for $\lambda>$ 0 , and $\Phi^{\prime}(\lambda)$ is completely monotonic on $(0, \infty)$; cf. Prüss [16] for these concepts, its properties, and implications for the evolutionary integral equation (3.1). For this class of kernels we have the following result.

Proposition 5. Suppose $a(t)$ is a completely positive function. Then $|\arg \widehat{a}(\lambda)| \leq$ $\pi / 2$ on $\Sigma_{\pi / 2}$, i.e. $\vartheta_{B} \leq \pi / 2$, and the resolvent kernels $r_{\mu}$ are subject to the estimates

$$
\left|r_{\mu}\right|_{1} \leq \frac{1}{\Phi_{0}+\operatorname{Re} \mu} \leq \frac{1}{\operatorname{Re} \mu}, \quad \text { for all } \mu \in \Sigma_{\pi / 2},
$$

and

$$
\left|t r_{\mu}\right|_{1} \leq \frac{\Phi_{1}}{\left(\Phi_{0}+\operatorname{Re} \mu\right)^{2}} \leq \frac{\Phi_{1}}{(\operatorname{Re} \mu)^{2}}, \quad \text { for all } \mu \in \Sigma_{\pi / 2},
$$

where

$$
\Phi_{0}=\Phi(0+)=\frac{1}{\widehat{a}(0+)} \quad \text { and } \quad \Phi_{1}=\Phi^{\prime}(0+)=\frac{-\widehat{a}^{\prime}(0+)}{\widehat{a}(0+)^{2}} .
$$

In particular, if $\Phi_{1}<\infty$, then (r) holds with $\beta=\delta$, for each $\varphi_{B}>\pi / 2$ and $\delta \in[0,1]$.

Proof. Consider $\mu>0$, first. It is well-known that the resolvent kernels $r_{\mu}(t)$ are nonnegative. Therefore,

$$
\left|r_{\mu}\right|_{1}=\int_{0}^{\infty} r_{\mu}(t) d t=\lim _{\varepsilon \rightarrow 0^{+}} \int_{0}^{\infty} e^{-\varepsilon t} r_{\mu}(t) d t=\lim _{\varepsilon \rightarrow 0^{+}} \widehat{r}_{\mu}(\varepsilon),
$$

and since $\widehat{r}_{\mu}(\lambda)=1 /(\Phi(\lambda)+\mu)$, we obtain

$$
\left|r_{\mu}\right|_{1}=\frac{1}{\Phi_{0}+\mu} \quad \text { for all } \mu>0 .
$$

This proves the first statement for positive $\mu$.

In the same way we obtain

$$
\left|t r_{\mu}\right|_{1}=\lim _{\varepsilon \rightarrow 0^{+}} \int_{0}^{\infty} t e^{-\varepsilon t} r_{\mu}(t) d t=-\lim _{\varepsilon \rightarrow 0^{+}} \widehat{r}_{\mu}^{\prime}(\varepsilon)=\frac{\Phi_{1}}{\left(\Phi_{0}+\mu\right)^{2}},
$$

which implies the second statement for $\mu>0$.

Now let $\mu=\mu_{0}+i \sigma$ be complex, $\mu_{0}>0$. Here we employ the propagation function $w(t ; \tau)$ associated with $a(t)$, which is defined via the relation

$$
\widehat{w}(\lambda ; \tau)=\frac{1}{\lambda} e^{-\Phi(\lambda) \tau}, \quad \text { for all } \lambda \in \Sigma_{\pi / 2}, \tau \geq 0 .
$$

It is well known that $w(t ; \tau)$ is nonnegative, and nondecreasing w.r.t. $t$; cf. Prüss [16]. The identity

$$
\widehat{r}_{\mu}(\lambda)=\int_{0}^{\infty} e^{-\mu \tau} e^{-\Phi(\lambda) \tau} d \tau
$$

then implies

$$
\int_{t}^{t+h} r_{\mu}(s) d s=\int_{0}^{\infty} e^{-\mu \tau}[w(t+h ; \tau)-w(t ; \tau)] d \tau, \quad t, h>0,
$$


and hence

$$
\begin{aligned}
\left|h^{-1} \int_{t}^{t+h} r_{\mu}(s) d s\right| & \leq h^{-1} \int_{0}^{\infty} e^{-\mu_{0} \tau}[w(t+h ; \tau)-w(t ; \tau)] d \tau \\
& =h^{-1} \int_{t}^{t+h} r_{\mu_{0}}(s) d s
\end{aligned}
$$

for all $t, h>0$. As $h \rightarrow 0^{+}$, the left hand side of this inequality converges to $\left|r_{\mu}\right|$ in $L_{\text {loc }}^{1}\left(\mathbb{R}_{+}\right)$while the right hand side approaches $r_{\mu_{0}}$ in $L^{1}\left(\mathbb{R}_{+}\right)$; therefore Lebesgue's theorem yields $r_{\mu} \in L^{1}\left(\mathbb{R}_{+}\right)$, and $\left|r_{\mu}\right|_{1} \leq\left|r_{\mu_{0}}\right|_{1}$. Thus the first statement holds for all $\mu \in \Sigma_{\pi / 2}$. In the same way we obtain also $\left|t r_{\mu}\right|_{1} \leq\left|t r_{\mu_{0}}\right|_{1}$ provided $\Phi_{1}$ is finite, and so the second claim follows as well.

The last statement is proved by Hölder's inequality. For this purpose let $\delta \in[0,1]$, and let $p=1 / \delta, 1 / p+1 / q=1$. Then

$$
\begin{aligned}
\left|t^{\delta} r_{\mu}\right|_{1} & \leq\left|t r_{\mu}\right|_{1}^{1 / p}\left|r_{\mu}\right|_{1}^{1 / q} \\
& \leq\left[\frac{\Phi_{1}}{\left(\Phi_{0}+\operatorname{Re} \mu\right)^{2}}\right]^{1 / p}\left[\frac{1}{\Phi_{0}+\operatorname{Re} \mu}\right]^{1 / q} \\
& =\frac{\Phi_{1}^{\delta}}{\left(\Phi_{0}+\operatorname{Re} \mu\right)^{1+\delta}}, \quad \text { for all } \mu \in \Sigma_{\pi / 2}, \delta \in[0,1] .
\end{aligned}
$$

This implies (r) with $\beta=\delta$.

Observe that condition (a) is not always satisfied when $a(t)$ is completely positive, since such kernels need not be 1-regular, in general. However, it was shown in Clément and Prüss [7] that the operator $B$ as given in Section 3 is still welldefined, and that it generates a $C_{0}$-semigroup of contractions in $L^{p}\left(\mathbb{R}_{+} ; Y\right)$ and admits bounded imaginary powers with power angle $\pi / 2$. Therefore the results of Section 3 still remain valid, provided $\Phi_{1}<\infty$. Completely monotonic kernels $a(t)$, in particular the kernels $a(t)=t^{\gamma-1}, \gamma \in(0,1]$, are completely positive. In particular, the considerations following Proposition 4 show that the condition $\Phi_{1}<\infty$ in Proposition 5 cannot be omitted.

3) The third class of kernels we want to consider here is motivated by the theory of viscoelasticity; cf. Prüss [16]. These kernels are of the form

$$
a(t)=a_{0}+a_{\infty} t+\int_{0}^{t} a_{1}(s) d s, \quad t>0,
$$

where $a_{0}, a_{\infty} \geq 0$, and $a_{1}(t)$ is 3 -monotone, i.e. nonnegative, nonincreasing, convex, $-\dot{a}(t)$ convex, with $\lim _{t \rightarrow \infty} a_{1}(t)=0$. Of course, we are only interested in the nontrivial case $a(t) \not \equiv 0$.

For kernels of the form (5.6) it can be shown that their Laplace transforms extend continuously to $\mathbb{C}_{+} \backslash\{0\}$ and that for the function

$$
g(\lambda)=a_{0}+a_{\infty} / \lambda+\widehat{a}_{1}(\lambda)
$$


the following estimates hold; cf. Prüss [16], Appendix to Section 3.

$$
\begin{gathered}
c_{2}\left[a_{0}+\int_{0}^{1 / \rho}-t \dot{a}_{1}(t) d t\right] \leq \operatorname{Re} g(i \rho) \leq C_{2}\left[a_{0}+\int_{0}^{1 / \rho}-t a_{1}(t) d t\right], \quad \rho>0 ; \\
(5.9) \quad c_{3}\left[\frac{a_{\infty}}{\rho}+\rho \int_{0}^{1 / \rho} t a_{1}(t) d t\right] \leq-\operatorname{Im} g(i \rho) \leq C_{3}\left[\frac{a_{\infty}}{\rho}+\rho \int_{0}^{1 / \rho} t a_{1}(t) d t\right] ; \\
(5.10) \quad\left|g^{(n)}(i \rho)\right| \leq C_{4}\left[\frac{a_{\infty}}{2 \rho^{n+1}}+\int_{0}^{1 / \rho} t^{n} a_{1}(t) d t\right], \quad \rho>0, n=0,1,2 .
\end{gathered}
$$

Here $c_{i}$ and $C_{i}$ are universal constants. These estimates are well-known as the SheaWainger estimates. Replacing $a_{\infty}+a_{1}(t)$ by $\left(a_{\infty}+a_{1}(t)\right) e^{-\sigma t}, \sigma \geq 0$, estimates (5.8), (5.7) and (5.10) show that $\operatorname{Re} g(\lambda)>0$ and

$$
\left|\lambda g^{\prime}(\lambda) / g(\lambda)\right| \leq C_{5}, \quad \text { for all } \operatorname{Re} \lambda \geq 0 .
$$

In particular $a(t)$ is 1-regular, and $\widehat{a}(\lambda)$ is never negative real nor zero, provided $a(t) \not \equiv a_{\infty} t$. Thus (a) holds with $\vartheta_{B} \leq \pi$. More precisely, estimates (5.8) and (5.9) yield $\vartheta_{B}<\pi$ if and only if

$$
\limsup _{t \rightarrow 0, \infty} \frac{t a_{\infty} / 2+t^{-1} \int_{0}^{t} s a_{1}(s) d s}{a_{0}+\int_{0}^{t}-s \dot{a}_{1}(s) d s}<\infty .
$$

This condition implies $a_{0}>0$ or $a_{1}(0+)=\infty$ (for the limit $t \rightarrow 0$ ), and $a_{\infty}=$ 0 (for the limit $t \rightarrow \infty$ ). It is implied by $a_{\infty}=0$ and $a_{1} \in L^{1}\left(\mathbb{R}_{+}\right)$, or by $a_{\infty}=0$ and $\liminf { }_{t \rightarrow \infty}-t \dot{a}_{1}(t) / a_{1}(t)>0$ (for $t \rightarrow \infty$ ), and by $a_{0}>0$, or by $\liminf \operatorname{in}_{t \rightarrow 0}-t \dot{a}_{1}(t) / a_{1}(t)>0$ (for $\left.t \rightarrow 0\right) . a_{1}(t)=t^{\gamma-1} / \Gamma(\gamma)$ with $\gamma \in(0,1)$ is a typical example with these properties.

We are now in position to state our result on kernels of the form (5.6).

Proposition 6. Let $a(t)$ be a kernel of the form (5.6) where $a_{0} \geq 0, a_{\infty}=0, a_{1}(t)$ 3-monotone with $\lim _{t \rightarrow \infty} a_{1}(t)=0$, and assume (5.12).

Then (a) is satisfied for some $\vartheta_{B}<\pi$ and the resolvent kernels $r_{\mu}$ are subject to the estimates

$$
\left|r_{\mu}\right|_{1} \leq \frac{C}{|\mu|}, \quad \text { for all } \mu \in \Sigma_{\pi-\varphi_{B}},
$$

and with $\rho_{0}=2 \vartheta_{B} / \pi$

$$
\left|t r_{\mu}\right|_{1} \leq \frac{C}{|\mu|^{1+1 / \rho_{0}}}, \quad \text { for all } \mu \in \Sigma_{\pi-\varphi_{B}},
$$

where $\varphi_{B}>\vartheta_{B}$ is arbitrary, and $C$ denotes a constant depending only on $\varphi_{B}$. Moreover,

$$
\left|t^{\delta} r_{\mu}\right|_{1} \leq \frac{C}{|\mu|^{1+\delta / \rho_{0}}}, \quad \text { for all } \mu \in \Sigma_{\pi-\varphi_{B}}
$$

for each $\delta \in[0,1]$.

Proof. The proof is based on Hardy's inequality (cf. Duren [12]), which is stated as 
Lemma 7. Suppose $f: \mathbb{C}_{+} \rightarrow \mathbb{C}$ is a bounded holomorphic function on the right half-plane such that $f^{\prime}$ belongs to the Hardy space $H^{1}\left(\mathbb{C}_{+}\right)$.

Then there is a function $b \in L^{1}\left(\mathbb{R}_{+}\right)$such that $\widehat{b}(\lambda)=f(\lambda)$ for all $\operatorname{Re} \lambda>0$, and $|b|_{L^{1}\left(\mathbb{R}_{+}\right)} \leq \frac{1}{2}\left|f^{\prime}\right|_{H^{1}\left(\mathbb{C}_{+}\right)}$.

For the Laplace transform of the resolvent kernel $r_{\mu}$ we obtain

$$
\widehat{r}_{\mu}(\lambda)=\frac{1}{\mu+1 / \widehat{a}(\lambda)}=\frac{g(\lambda)}{\lambda+\mu g(\lambda)}, \quad \operatorname{Re} \lambda>0 .
$$

By assumption (5.12) we have $\vartheta_{B}<\pi$. Fix any $\varphi_{B} \in\left(\vartheta_{B}, \pi\right)$; then there is a constant $C>0$ such that

$$
|\lambda+\mu g(\lambda)| \geq C^{-1}[|\lambda|+|\mu||g(\lambda)|], \quad \text { for all } \operatorname{Re} \lambda \geq 0, \mu \in \Sigma_{\pi-\varphi_{B}} .
$$

This implies

$$
\left|\widehat{r}_{\mu}(\lambda)\right| \leq C \frac{|g(\lambda)|}{|\lambda|+|\mu||g(\lambda)|} \leq \frac{C}{|\mu|}, \quad \text { for all } \operatorname{Re} \lambda \geq 0, \mu \in \Sigma_{\pi-\varphi_{B}},
$$

which means that $h_{\mu}=\widehat{r}_{\mu}$ is bounded and holomorphic on the right half-plane. For the derivative of $h_{\mu}$ we obtain

$$
h_{\mu}^{\prime}=\frac{\lambda g^{\prime}(\lambda)-g(\lambda)}{(\lambda+\mu g(\lambda))^{2}} .
$$

Hence by (5.11) we obtain

$$
\left|h_{\mu}^{\prime}(\lambda)\right| \leq C^{2}\left(1+C_{5}\right) \frac{|g(\lambda)|}{(|\lambda|+|\mu||g(\lambda)|)^{2}},
$$

and therefore

$$
\begin{aligned}
\left|h_{\mu}^{\prime}\right|_{H^{1}\left(\mathbb{C}_{+}\right)} & =\int_{-\infty}^{\infty}\left|h_{\mu}^{\prime}(i \rho)\right| d \rho \leq C_{6} \int_{-\infty}^{\infty} \frac{|g(i \rho)|}{(|\rho|+|\mu||g(i \rho)|)^{2}} d \rho \\
& \leq C_{7} \int_{0}^{\infty} \frac{a(1 / \rho)}{(\rho+|\mu| a(1 / \rho))^{2}} d \rho=C_{7} \int_{0}^{\infty} \frac{a(s)}{(1+s|\mu| a(s))^{2}} d s
\end{aligned}
$$

where we employed estimates (5.7). The function $a(s)$ is nondecreasing, which implies the inequality

$$
\frac{d}{d s}(s a(s))=a(s)+s \dot{a}(s) \geq a(s), \quad s>0,
$$

and therefore

$$
\begin{aligned}
\left|h_{\mu}^{\prime}\right|_{H^{1}\left(\mathbb{C}_{+}\right)} & \leq C_{7} \int_{0}^{\infty} \frac{a(s)}{(1+s|\mu| a(s))^{2}} d s \\
& \leq C_{7} \int_{0}^{\infty} \frac{d(s a(s)) / d s}{(1+|\mu| s a(s))^{2}} d s \\
& =C_{7} \int_{0}^{\infty} \frac{1}{(1+|\mu| r)^{2}} d r=\frac{C_{7}}{|\mu|} .
\end{aligned}
$$

By uniqueness of the Laplace transform, Lemma 7 then implies $r_{\mu} \in L^{1}\left(\mathbb{R}_{+}\right)$and $\left|r_{\mu}\right|_{1} \leq C_{7} / 2|\mu|$, for all $\mu \in \Sigma_{\pi-\varphi}$. This proves (5.13).

To prove the second estimate (5.14) we proceed similarly. It is easy to see that $h_{\mu}^{\prime}$ is bounded on $\mathbb{C}_{+}$, and by uniqueness of the Laplace transform we have 
$h_{\mu}^{\prime}(\lambda)=-\widehat{t r}_{\mu}(\lambda)$ on the open right half-plane. Therefore, by Lemma 7 it is sufficient to estimate $h_{\mu}^{\prime \prime}$ in the Hardy space $H^{1}\left(\mathbb{C}_{+}\right)$. We have

$$
h_{\mu}^{\prime \prime}(\lambda)=\frac{\lambda g^{\prime \prime}(\lambda)}{(\lambda+\mu g(\lambda))^{2}}-2\left[\frac{\lambda g^{\prime}(\lambda)}{g(\lambda)}-1\right] \frac{g(\lambda)+\mu g(\lambda) g^{\prime}(\lambda)}{\left(\lambda+\mu g(\lambda)^{3}\right.}
$$

hence (5.11) and (5.16) yield

$$
\left|h_{\mu}^{\prime \prime}(\lambda)\right| \leq C_{8} \frac{\left|\lambda g^{\prime \prime}(\lambda)\right|+\left|g^{\prime}(\lambda)\right|+1 /|\mu|}{(|\lambda|+|\mu||g(\lambda)|)^{2}} .
$$

Therefore, with $\psi(s)=\int_{0}^{s} t a_{1}(t) d t$ and with (5.10) we obtain

$$
\left|\rho g^{\prime \prime}(i \rho)\right| \leq C_{4}|\rho| \int_{0}^{1 /|\rho|} t^{2} a_{1}(t) d t \leq C_{4} \psi(1 /|\rho|) .
$$

This then implies

$$
\begin{aligned}
\left|h_{\mu}^{\prime \prime}\right|_{H^{1}\left(\mathbb{C}_{+}\right)} & =\int_{-\infty}^{\infty}\left|h_{\mu}^{\prime \prime}(i \rho)\right| d \rho \leq C_{9} \int_{0}^{\infty} \frac{\psi(1 / \rho)+1 /|\mu|}{(\rho+|\mu| a(1 / \rho))^{2}} d \rho \\
& =C_{9}\left[\int_{0}^{\infty} \frac{\psi(s)}{(1+|\mu| s a(s))^{2}} d s+|\mu|^{-1} \int_{0}^{\infty} \frac{1}{(1+|\mu| s a(s))^{2}} d s\right] \\
& =C_{9}\left[I_{1}+I_{2} /|\mu|\right] .
\end{aligned}
$$

Hence it remains to obtain bounds for the integrals $I_{1}$ and $I_{2}$.

(i) To estimate the first part of $I_{1}$, observe that $\psi(s) \leq s a(s) \leq s d(s a(s)) / d s$; therefore an integration by parts and $a(s) \geq s \dot{a}(s)$ yield, with some $\varepsilon>0$ which will be chosen later,

$$
\begin{aligned}
\int_{0}^{\varepsilon} \frac{\psi(s)}{(1+|\mu| s a(s))^{2}} d s & \leq \int_{0}^{\varepsilon} \frac{d(s a(s)) / d s}{(1+|\mu| s a(s))^{2}} s d s \\
& =-\left.\frac{s}{|\mu|(1+|\mu| s a(s))}\right|_{0} ^{\varepsilon}+\int_{0}^{\varepsilon} \frac{1}{|\mu|(1+|\mu| s a(s))} d s \\
& \leq \frac{\varepsilon}{|\mu|} .
\end{aligned}
$$

(ii) The remaining part of $I_{1}$ is estimated also via an integration by parts as follows:

$$
\begin{aligned}
\int_{\varepsilon}^{\infty} \frac{\psi(s)}{(1+|\mu| s a(s))^{2}} d s & \leq \int_{\varepsilon}^{\infty} \frac{\psi(s)}{(|\mu| a(s))^{2}} \frac{d s}{s^{2}} \\
& =-\left.\frac{\psi(s)}{s|\mu|^{2} a(s)^{2}}\right|_{\varepsilon} ^{\infty}+\int_{\varepsilon}^{\infty} \frac{\dot{\psi}(s) a(s)-2 \psi(s) \dot{a}(s)}{|\mu|^{2} a(s)^{3} s} d s \\
& \leq \frac{\psi(\varepsilon)}{\varepsilon|\mu|^{2} a(\varepsilon)^{2}}+\int_{\varepsilon}^{\infty} \frac{s \dot{a}(s)}{s|\mu|^{2} a(s)^{2}} d s \\
& =\frac{\psi(\varepsilon) / \varepsilon+a(\varepsilon)}{|\mu|^{2} a(\varepsilon)^{2}} \leq \frac{2}{|\mu|^{2} a(\varepsilon)} .
\end{aligned}
$$

Combining (i) and (ii), we arrive at

$$
I_{1} \leq \frac{\varepsilon}{|\mu|}\left[1+\frac{2}{|\mu| \varepsilon a(\varepsilon)}\right], \quad \text { for all } \mu \in \Sigma_{\pi-\varphi_{B}} .
$$


(iii) To obtain an appropriate bound for $I_{2}$ we proceed as follows:

$$
\begin{aligned}
I_{2} & =\int_{0}^{\infty} \frac{1}{(1+|\mu| s a(s))^{2}} d s \leq \varepsilon+\int_{\varepsilon}^{\infty} \frac{1}{(|\mu| s a(s))^{2}} d s \\
& \leq \varepsilon+\frac{1}{|\mu|^{2} a(\varepsilon)^{2}} \int_{\varepsilon}^{\infty} \frac{d s}{s^{2}}=\varepsilon\left[1+\frac{1}{|\mu|^{2}(\varepsilon a(\varepsilon))^{2}}\right] .
\end{aligned}
$$

As $s$ runs from 0 to $\infty$, the function $s \mapsto s a(s)$ is strictly increasing from 0 to $\infty$; therefore there is a unique value of $s$, say $\varepsilon>0$, such that $\varepsilon a(\varepsilon)=1 /|\mu|$. This implies $I_{1} \leq 3 \varepsilon /|\mu|$ and $I_{2} \leq 2 \varepsilon$; hence

$$
\left|h_{\mu}^{\prime \prime}\right|_{H^{1}\left(\mathbb{C}_{+}\right)} \leq 5 C_{9} \frac{\varepsilon}{|\mu|} .
$$

To estimate $\varepsilon$ in terms of $|\mu|$ we employ (5.7) and (3.6):

$$
\begin{aligned}
|\mu|^{-1} & =\varepsilon a(\varepsilon) \geq c_{1}|g(i / \varepsilon)| \varepsilon=c_{1}|\widehat{a}(i / \varepsilon)| \\
& \geq c_{2} \widehat{a}(1 / \varepsilon) \geq c_{3} \varepsilon^{\rho_{0}}
\end{aligned}
$$

hence $\varepsilon \leq c_{4}|\mu|^{-1 / \rho_{0}}$. This completes the proof of (5.14). The last statement follows from interpolation, as in the proof of Proposition 5.

\section{Application to Parabolic Partial Differential and InTEGRo-DiffERENTIAL EQUATIONS}

In this section we want to apply Theorem 2 and Corollary 3 to parabolic partial differential and integro-differential equations of second order in space. For this purpose, let $\Omega \subset \mathbb{R}^{n}$ be a bounded domain with boundary $\partial \Omega$ of class $C^{2}$. Consider the problem

$$
\left\{\begin{array}{c}
u(t, x)+\int_{0}^{t} a(t-s)\{\nu u(s, x)-\operatorname{div}[b(s, x) \nabla u(s, x)]-f(s, x)\} d s=0 \\
t \geq 0, x \in \Omega \\
n(x) \cdot(b(t, x) \nabla u(t, x))=0, \quad t \geq 0, x \in \Gamma_{1} \\
u(t, x)=0, \quad t \geq 0, x \in \Gamma_{0}
\end{array}\right.
$$

where $\Gamma_{j} \subset \partial \Omega$ are open and closed in $\partial \Omega$, such that $\Gamma_{1} \cap \Gamma_{0}=\emptyset, \Gamma_{1} \cup \Gamma_{0}=\partial \Omega$, and $n(x)$ denotes the outer normal of $\Omega$ at $x \in \partial \Omega$. Denoting the space of symmetric real $n \times n$ matrices by $\operatorname{Sym}(n), b: \mathbb{R}_{+} \times \bar{\Omega} \rightarrow \operatorname{Sym}(n)$ is assumed at least to be continuous with bounded partial derivatives w.r.t. $x \in \Omega$, and to be uniformly positive definite in the sense that there exists a constant $b_{0}>0$ such that

$$
b_{0}|\xi|^{2} \leq \xi \cdot b(t, x) \xi \leq b_{0}^{-1}|\xi|^{2} \quad \text { for all } t \geq 0, x \in \Omega, \xi \in \mathbb{R}^{n} .
$$

Concerning the kernel $a(t)$ we assume that it belongs to one of the three classes studied in Section 5 , in particular, $a(t)=1$ arises as a special case; this case corresponds to the boundary value problem

$$
\left\{\begin{array}{l}
u_{t}(t, x)+\nu u(t, x)=\operatorname{div}[b(t, x) \nabla u(t, x)]-f(t, x), \quad t \geq 0, x \in \Omega, \\
n(x) \cdot(b(t, x) \nabla u(t, x))=0, \quad t \geq 0, x \in \Gamma_{1}, \\
u(t, x)=0, \quad t \geq 0, x \in \Gamma_{0}, \quad u(0, x)=0, \quad x \in \Omega
\end{array}\right.
$$

The family $\{L(t)\}_{t \geq 0}$ will be the $L^{q}$-realizations of the underlying elliptic boundary value problems; more precisely, we define

$$
\left\{\begin{array}{l}
(L(t) u)(x)=-\operatorname{div}[b(t, x) \nabla u(x)], \quad t \geq 0, x \in \Omega, \\
D(L(t))=\left\{u \in H^{2, q}(\Omega):\left.u\right|_{\Gamma_{0}}=0,\left.n \cdot b(t, \cdot) \nabla u\right|_{\Gamma_{1}}=0\right\},
\end{array}\right.
$$


where the boundary values of $u$ and $\nabla u$ are understood in the sense of traces. Thus as the base space $Y$ we choose $Y=L^{q}(\Omega)$ with $1<q<\infty$, which is well-known to be of class $\mathcal{H} \mathcal{T}$. Then (6.1) and (6.3) written in abstract form become (3.1) and (3.2), respectively. Observe that in case $\Gamma_{1} \neq \emptyset$ and $b$ is not constant in time, the domains $D(L(t))$ are not constant. The norm in $H^{s, q}(\Omega)$ will be denoted by $|\cdot|_{s, q}$, and that of $L^{q}(\Omega)$ by $|\cdot|_{q}$.

It is well-known (see e.g. Lunardi [15], Chap. 3) that the operators $L(t)$ are sectorial with spectral angle $\phi_{L(t)}=0$, and for each $\varphi_{A} \in(0, \pi)$ there is a constant $M_{A}>0$ such that

$$
\left|(\lambda+L(t))^{-1}\right| \leq \frac{M_{A}}{1+|\lambda|}, \quad t \geq 0, \lambda \in \Sigma_{\pi-\varphi_{A}},
$$

and

$$
\left|L(t)^{-1} g\right|_{2, q} \leq M_{A}^{\prime}|g|_{q}, \quad t \geq 0, g \in L^{q}(\Omega),
$$

except for the case of the pure Neumann problem $\Gamma_{0}=0$, where the kernels of $L(t)$ are nontrivial. For the sake of simplicity we exclude this case and assume from now on that $\Gamma_{0} \neq \emptyset$.

If $q=2$ it is also well-known that $L(t)$ is selfadjoint and positive definite, hence admits bounded imaginary powers and

$$
\left|L(t)^{i s}\right|_{2}=1, \quad \text { for all } t \geq 0, s \in \mathbb{R} ;
$$

cf. e.g. Prüss and Sohr [17], Example 1.

If $q \neq 2$ but $\Gamma_{1}=\emptyset$, it has been shown in Prüss and Sohr [18] that $L(t)$ admits bounded imaginary powers in $L^{q}(\Omega)$ and that for each $\varphi_{A} \in(0, \pi)$ there is a constant $K_{A}>0$ such that

$$
\left|L(t)^{i s}\right|_{q}=K_{A} e^{\varphi_{A}|s|}, \quad \text { for all } t \geq 0, s \in \mathbb{R} ;
$$

in particular $\theta_{L(t)}=0$.

If $q \neq 2$ and $\Gamma_{1}$ is arbitrary, Duong [10] obtained $L(t) \in B I P\left(L^{q}(\Omega)\right)$ and

$$
\left|L(t)^{i s}\right|_{q}=K_{A} e^{|s| \pi / 2}, \quad \text { for all } t \geq 0, s \in \mathbb{R} .
$$

By interpolation with (6.7) this estimate can be improved to $\theta_{L(t)} \leq|1-2 / q| \pi / 2$, and for each $\varphi_{A}>|1-2 / q| \pi / 2$ there is a constant $K_{A}>0$ such that (6.8) is valid. More recently Duong and Robinson [11] claim (6.8) for each $\varphi_{A}>0$. This completes the verification of $(\mathbf{L})$ for the class of operator families $\{L(t)\}_{t \geq 0}$ given by (6.4).

To verify the commutator condition (C) we use an argument which is similar to that employed by Acquistapace [1]. Let a function $g \in L^{q}(\Omega)$ be given, and define $f=(\lambda+L(s)) L(s)^{-1} g, u=(\lambda+L(t))^{-1} f$, and $v=(\lambda+L(s))^{-1} f$. Then with the notation $\mathcal{L}(t)=-\operatorname{div}[b(t, \cdot) \nabla]$ and $\mathcal{B}(t)=n \cdot[b(t, \cdot) \nabla]$ we have

$$
\left\{\begin{array}{cc}
\lambda u+\mathcal{L}(t) u=f & \text { on } \Omega \\
\mathcal{B}(t) u=0 & \text { on } \Gamma_{1} \\
u=0 & \text { on } \Gamma_{0}
\end{array}\right\} \quad \text { and } \quad\left\{\begin{array}{cc}
\lambda v+\mathcal{L}(s) v=f & \text { on } \Omega \\
\mathcal{B}(s) v=0 & \text { on } \Gamma_{1} \\
v=0 & \text { on } \Gamma_{0}
\end{array}\right\} \text {. }
$$

Therefore $w=u-v$ solves the boundary value problem

$$
\left\{\begin{array}{ccc}
\lambda w+\mathcal{L}(t) w=(\mathcal{L}(s)-\mathcal{L}(t)) v & \text { on } & \Omega \\
\mathcal{B}(t) w=(\mathcal{B}(s)-\mathcal{B}(t)) v & \text { on } & \Gamma_{1} \\
w=0 & \text { on } & \Gamma_{0}
\end{array}\right.
$$


and hence can be written as

$$
w=(\lambda+L(t))^{-1}(\mathcal{L}(s)-\mathcal{L}(t)) v+S_{\lambda}(t)(\mathcal{B}(s)-\mathcal{B}(t)) v,
$$

where the so-called Dirichlet map $S_{\lambda}(t)$ is defined according to

$$
S_{\lambda}(t) \varphi=z \quad \Leftrightarrow \quad\left\{\begin{array}{cc}
\lambda z+\mathcal{L}(t) z=0 & \text { on } \Omega, \\
\mathcal{B}(t) z=\varphi & \text { on } \Gamma_{1}, \\
z=0 & \text { on } \Gamma_{0} .
\end{array}\right.
$$

Since $v=(\lambda+L(s))^{-1} f=L(s)^{-1} g$ we finally obtain the following representation for the commutator $C(t, s, \lambda)=L(t)(\lambda+L(t))^{-1}\left[L(t)^{-1}-L(s)^{-1}\right]$ :

$$
C(t, s, \lambda) g=(\lambda+L(t))^{-1}(\mathcal{L}(s)-\mathcal{L}(t)) L(s)^{-1} g+S_{\lambda}(t)(\mathcal{B}(s)-\mathcal{B}(t)) L(s)^{-1} g,
$$

for all $t, s \geq 0, \lambda \in \Sigma_{\pi-\varphi_{A}}$. The first term in this representation is estimated easily. In fact, suppose $b(\cdot, x)$ and $b_{x_{j}}(\cdot, x)$ belong to $C^{\delta}\left(\mathbb{R}_{+}\right)$uniformly w.r.t. $x \in \Omega$, for some $\delta>0$. Then

$$
|(\mathcal{L}(s)-\mathcal{L}(t)) u|_{q} \leq C_{1}|t-s|^{\delta}|u|_{2, q}, \quad t, s \geq 0, u \in H^{2, q}(\Omega) .
$$

By (6.5) this implies

$$
\left|(\lambda+L(t))^{-1}(\mathcal{L}(s)-\mathcal{L}(t)) L(s)^{-1} g\right|_{q} \leq \frac{M_{A}}{1+|\lambda|} C_{1}|t-s|^{\delta} M_{A}^{\prime}|g|_{q},
$$

for all $t, s \geq 0$, and $g \in L^{q}(\Omega)$. Concerning the second term on the right hand side of (6.11), we first refer to Triebel [21] for the following estimate of the Dirichlet map:

$$
\left|S_{\lambda}(t) \varphi\right|_{q} \leq C_{q}\left[\frac{|\widetilde{\varphi}|_{q}}{(1+|\lambda|)^{1 / 2}}+\frac{|\nabla \widetilde{\varphi}|_{q}}{1+|\lambda|}\right],
$$

where $\widetilde{\varphi}$ denotes any extension of $\varphi$ to $\Omega$ in the sense that $\widetilde{\varphi}=\varphi$ on $\Gamma_{1} ; C_{q}$ is independent of $t \geq 0$. Fix any extension $\widetilde{n}$ of the outer normal field $n(x)$ to $\Omega$ which is of class $C^{1}$. Then by (6.13) we have

$$
\begin{aligned}
\left|S_{\lambda}(t)(\mathcal{B}(s)-\mathcal{B}(t)) L(s)^{-1} g\right|_{q} \leq C_{q}[ & (1+|\lambda|)^{-1 / 2}\left|(\widetilde{\mathcal{B}}(s)-\widetilde{\mathcal{B}}(t)) L(s)^{-1} g\right|_{q} \\
& \left.+(1+|\lambda|)^{-1}\left|\nabla(\widetilde{\mathcal{B}}(s)-\widetilde{\mathcal{B}}(t)) L(s)^{-1} g\right|_{q}\right],
\end{aligned}
$$

where $\widetilde{\mathcal{B}}(t)=\widetilde{n}(x) \cdot(b(t, x) \nabla)$. Then

$$
\begin{aligned}
\left|(\widetilde{\mathcal{B}}(s)-\widetilde{\mathcal{B}}(t)) L(s)^{-1} g\right|_{q} & \leq|\widetilde{n}|_{\infty} \sup _{x \in \Omega}|b(t, x)-b(s, x)|\left|\nabla L(s)^{-1} g\right|_{q} \\
& \leq|\widetilde{n}|_{\infty}|b|_{\delta}|t-s|^{\delta} M_{A}^{\prime}|g|_{q} \\
& =C_{2}|t-s|^{\delta}|g|_{q}
\end{aligned}
$$

where $|b|_{\delta}=\sup \left\{|b(t, x)-b(s, x)||t-s|^{-\delta}: t, s \geq 0, t \neq s, x \in \Omega\right\}$. Similarly, if $b_{x_{j}}$ are also uniformly Hölder-continuous in $t$, we obtain

$$
\begin{aligned}
\left|\nabla(\widetilde{\mathcal{B}}(s)-\widetilde{\mathcal{B}}(t)) L(s)^{-1} g\right|_{q} \leq & |\widetilde{n}|_{\infty} \sup _{x \in \Omega}|b(t, x)-b(s, x)|\left|\nabla^{2} L(s)^{-1} g\right|_{q} \\
& +|\widetilde{n}|_{\infty} \sup _{x \in \Omega}|\nabla \cdot(b(t, x)-b(s, x))|\left|\nabla L(s)^{-1} g\right|_{q} \\
& +|\nabla \cdot \widetilde{n}|_{\infty} \sup _{x \in \Omega}|b(t, x)-b(s, x)|\left|\nabla L(s)^{-1} g\right|_{q} \\
\leq & C_{3}|t-s|^{\delta}|g|_{q} .
\end{aligned}
$$


Therefore

$$
\begin{aligned}
\left|S_{\lambda}(t)(\mathcal{B}(s)-\mathcal{B}(t)) L(s)^{-1} g\right|_{q} & \leq C_{q}\left[\frac{C_{2}}{(1+|\lambda|)^{1 / 2}}+\frac{C_{3}}{1+|\lambda|}\right]|t-s|^{\delta}|g|_{q} \\
& \leq \frac{C_{4}|t-s|^{\delta}}{(1+|\lambda|)^{1 / 2}}|g|_{q} .
\end{aligned}
$$

Combining estimates (6.12) and (6.14), we arrive at

$$
|C(t, s, \lambda)|_{q} \leq C_{5} \frac{|t-s|^{\delta}}{(1+|\lambda|)^{1 / 2}}, \quad \text { for all } t, s \geq 0, \lambda \in \Sigma_{\pi-\varphi_{A}},
$$

which shows that the commutator condition (C) holds with $\alpha=1 / 2, \varphi_{A}>0$ arbitrary, and $\delta>0$, provided $b(\cdot, x), b_{x_{j}}(\cdot, x) \in C^{\delta}\left(\mathbb{R}_{+}\right)$uniformly for $x \in \Omega$. Observe that for the case of Dirichlet boundary conditions $\Gamma_{1}=\emptyset, \alpha$ can be chosen as $\alpha=0$.

We are now in position to apply Theorem 2 and Corollary 3 and obtain the following result.

Theorem 3. Let $\Omega \subset \mathbb{R}^{n}$ be a bounded domain with boundary $\partial \Omega$ of class $C^{2}$, $\Gamma_{j} \subset \partial \Omega$ open and closed in $\partial \Omega$ such that $\Gamma_{1} \cap \Gamma_{0}=\emptyset, \Gamma_{1} \cup \Gamma_{0}=\partial \Omega$, and $\Gamma_{0} \neq \emptyset$. Assume that $b: \mathbb{R}_{+} \times \bar{\Omega} \rightarrow \operatorname{Sym}(n)$ satisfies the strong ellipticity condition (6.2) and $b, b_{x_{j}} \in C^{\delta}\left(\mathbb{R}_{+} ; C(\bar{\Omega})\right)$, for some $\delta>0$. Let $p, q \in(1, \infty), J=[0, T]$, or $J=\mathbb{R}_{+}$ but $\nu>0$ sufficiently large.

Then for every $f \in L^{p}\left(J ; L^{q}(\Omega)\right)$, problem (6.1) admits a unique solution $u \in$ $L^{p}\left(J ; H^{2, q}(\Omega)\right)$, provided the kernel a(t) satisfies one of the following conditions:

(i) $a(t)=t^{\gamma-1} / \Gamma(\gamma), \gamma \in(0,2), \delta>\gamma / 2$.

(ii) $a(t)$ is completely positive, $-\widehat{a}^{\prime}(0+) / \widehat{a}(0+)^{2}<\infty, \delta>1 / 2$.

(iii) $a(t)$ is as in Proposition 6 and $\delta>\rho_{0} / 2$.

In addition, if $\lim \sup _{r \rightarrow \infty}|\widehat{a}(r)| r^{\rho}<\infty$, then the solution $u$ of (3.1) belongs to $H_{0}^{\rho, p}\left(J ; L^{q}(\Omega)\right)$.

It is clear from the derivations in this section that Theorem 3 can be generalized to other types of elliptic operators $\mathcal{L}(t)$ if only the relevant estimates for the resolvent, for the Dirichlet map, and for the imaginary powers of $\mathcal{L}(t)$ are valid, and the parabolicity condition is satisfied. These subjects as well as applications to quasilinear problems will be taken up in the near future.

\section{REFERENCES}

1. P. Acquistapace. Evolution operators and strong solutions of abstract linear parabolic equations. Diff. Int. Equations, 1:433-457, 1988. MR 90b:34094

2. P. Acquistapace and B. Terreni. A unified approach to abstract linear non-autonomous parabolic equations. Rend. Sem. Mat. Univ. Padova, 78:47-107, 1987. MR 89e:34099

3. J.B. Baillon and Ph. Clément. Examples of unbounded imaginary powers of operators. J. Funct. Anal., 100:419-434, 1991. MR 92j:47036

4. J. Bourgain. Some remarks on Banach spaces in which martingale difference sequences are unconditional. Ark. Mat., 22:163-168, 1983. MR 85a:46011

5. J. Bourgain. Vector-valued singular integrals and the $H^{1}-B M O$ duality. In D. Burkholder, editor, Probability Theory and Harmonic Analysis, pages 1-19, New-York, 1986. Marcel Dekker. MR 87j:42049b

6. D.L. Burkholder. Martingales and Fourier analysis in Banach spaces. In G. Letta and M. Pratelli, editors, Probability and Analysis, volume 1206 of Lect. Notes Math., pages 61-108, Berlin, 1986. Springer Verlag. MR 88c:42017

7. Ph. Clément and J. Prüss. Completely positive measures and Feller semigroups. Math. Ann., 287:73-105, 1990. MR 91d:45011 
8. G. Da Prato and P. Grisvard. Sommes d'opérateurs linéaires et équations différentielles opérationelles. J. Math. Pures Appl., 54:305-387, 1975. MR 56:1129

9. G. Dore and A. Venni. On the closedness of the sum of two closed operators. Math. Z., 196:189-201, 1987. MR 88m:47072

10. X. T. Duong. $H_{\infty}$ - functional calculus for second order elliptic partial differential operators on $L^{p}$-spaces. In I. Doust, B. Jefferies, C. Li, and A. McIntosh, editors, Operators in Analysis, pages 91-102, Sydney, 1989. Australian National University. MR 91m:35072

11. X. T. Duong and D. W. Robinson. Gaussian bounds, Brownian estimates and $H_{\infty}$-functional calculus. Preprint, 1994.

12. P.L. Duren. Theory of $H^{p}$ Spaces, volume 38 of Pure Appl. Math. Acad. Press, New York, 1970. MR 42:3552

13. M. Fuhrmann. Sums of linear operators of parabolic type : a priori estimates and strong solutions. Ann. Mat. Pura Appl., 164:229-257, 1993.

14. R. Labbas and B. Terreni. Somme d'opérateurs linéaires de type parabolique. Boll. Un. Mat. Ital., (7) 1-B:545-569, 1987. MR 89g:47016

15. A. Lunardi. Analytic Semigroups and Optimal Regularity in Parabolic Problems. BirkhäuserVerlag, Basel, 1995. MR 96e:47039

16. J. Prüss. Evolutionary Integral Equations and Applications. Birkhäuser Verlag, Basel, 1993. MR 94h: 45010

17. J. Prüss and H. Sohr. On operators with bounded imaginary powers in Banach spaces. Math. Z., 203:429-452, 1990. MR 91b:47030

18. J. Prüss and H. Sohr. Boundedness of imaginary powers of second-order elliptic differential operators in $L^{p}$. Hiroshima Math. J., 23:161-192, 1993. MR 94d:47051

19. P.E. Sobolevskii. On equations of parabolic type. Amer. Math. Soc. Transl., 49:1-62, 1965. MR 25:5297

20. H. Tanabe. Equations of Evolution, volume 6 of Monographs and Studies in Mathematics. Pitman, London, 1979. MR 82g:47032

21. H. Triebel. Interpolation Theory, Function Spaces, Differential Operators. North Holland, Amsterdam, 1978. MR 80i: 46032

22. F. Zimmermann. On vector-valued Fourier multiplier theorems. Studia Math., 93:201-222, 1989. MR 91b:46031

Mathematik V, Universität Ulm, D-89069 Ulm, Germany

E-mail address: monniaux@mathematik.uni-ulm.de

Fachbereich Mathematik und Informatik, Martin-Luther- Universität Halle-WittenBerg, Theodor-Lieser-Str. 5, D-06120 Halle, Germany

E-mail address: anokd@volterra.mathematik.uni-halle.de 